\title{
The Accurate Numerical Solution of Highly Oscillatory Ordinary Differential Equations*
}

\author{
By Robert E. Scheid, Jr.
}

\begin{abstract}
An asymptotic theory for weakly nonlinear, highly oscillatory systems of ordinary differential equations leads to methods which are suitable for accurate computation with large time steps. The theory is developed for systems of the form

$$
\begin{aligned}
& \mathbf{Z}^{\prime}=(A(t) / \varepsilon) \mathbf{Z}+\mathbf{H}(\mathbf{Z}, t), \\
& \mathbf{Z}(0, \varepsilon)=\mathbf{Z}_{0}, \quad 0<t<T, 0<\varepsilon \ll 1,
\end{aligned}
$$

where the diagonal matrix $A(t)$ has smooth, purely imaginary eigenvalues and the components of $\mathbf{H}(\mathbf{Z}, t)$ are polynomial in the components of $\mathbf{Z}$ with smooth $t$-dependent coefficients. Computational examples are presented.
\end{abstract}

1. Introduction. Mathematical modeling of a chemical, electrical, mechanical or biological process often leads to a differential system whose Jacobian has at least one eigenvalue with either a large negative real part or a large imaginary part. Even when the underlying structure is quite complicated, one generally can analyze the stiffness of such a system through the simple scalar equation:

$$
\begin{aligned}
& d y / d t=a y, \quad t \geqslant 0, \\
& y(0)=y_{0}, \\
& \text { Case I: } \operatorname{Re}\{-a\} \gg 1, \\
& \text { Case II: }|\operatorname{Im}\{a\}| \gg 1 .
\end{aligned}
$$

Unless one is prepared to compute with an excessively small time step, most conventional numerical methods are ill-suited to the problem for reasons of stability or accuracy. For example, in Table 1 we consider several generic schemes as applied to the system (1.1) with mesh width $h$.

On considering the stiff limit $(|a h| \rightarrow \infty$ with $\operatorname{Re}\{a h\} \leqslant 0)$, we find that the first method is unstable while the second and third are stable. Moreover, the solution of $\langle 1 b\rangle$ decays rapidly on the grid points, while the solution of $\langle 1 \mathrm{c}\rangle$ can be characterized as grid oscillations. These observations do not contradict the general theory which has been developed for the nonstiff limit $(|a h| \rightarrow 0)$ but rather indicate that one cannot expect convergence in the stiff limit.

Received July 30, 1982.

1980 Mathematics Subject Classification. Primary 65L05, 34E15.

Key words and phrases. Oscillatory, numerical solution of ordinary differential equations, stiff equations.

${ }^{*}$ Research supported by the Office of Naval Research under Contract No. N00014-80-C-0076. 


\begin{tabular}{llll}
\hline METHOD & FORMULATION/SOLUTION & $|a h| \rightarrow \infty$ & $(\operatorname{Re}\{a h\} \leqslant 0)$ \\
\hline $\begin{array}{l}\text { Forward } \\
\text { Euler }\end{array}$ & $\left\{\begin{array}{l}v_{N+1}=v_{N}(1+a h) \\
v_{0}=y_{0} \\
v_{N}=(1+a h)^{N} v_{0}\end{array}\right.$ & $\left|v_{N}\right| \rightarrow \infty$ & $(N>0)$ \\
Backward & $\left\{\begin{array}{l}v_{N+1}(1-a h)=v_{N} \\
v_{0}=y_{0} \\
\text { Euler }\end{array}\right.$ & & \\
$\langle$ lb $\rangle$ & $v_{N}=[1 /(1-a h)]^{N} v_{0}$ & $\left|v_{N}\right| \rightarrow 0$ & $(N>0)$ \\
$\begin{array}{l}\text { Trapezoidal } \\
\text { Rule }\end{array}$ & $\left\{\begin{array}{l}v_{N+1}(1-a h / 2)=v_{N}(1+a h / 2) \\
v_{0}=y_{0}\end{array}\right.$ & & \\
$\langle 1 \mathrm{c}\rangle$ & $v_{N}=[(1+a h) /(1-a h)]^{N} v_{0}$ & & \\
\hline
\end{tabular}

Nevertheless, for Case I the solution of $\langle 1 \mathrm{~b}\rangle$ is qualitatively similar to the solution of the differential equation (1.1). Much has been made of this salient feature of the backwards Euler formulation, and many schemes with similar stability properties have been proposed for stiff problems of this type (see, for example, Lambert [15] and Kreiss [13]). With the exception of a thin boundary layer, such problems have nicely behaved solutions. Our aim is a detailed numerical analysis of the highly oscillatory case (II), in which the rapid changes are expected to persist.

Since the fundamental work of Poincaré, mathematicians studying oscillatory phenomena have developed an extensive arsenal of perturbative techniques including multiscaling, averaging, and the near-identity transformation (see, for example, Kevorkian and Cole [12], Nayfeh [24], and Neu [23]). For the most part, these tools are difficult to implement numerically since the analytic manipulations require a competence not to be expected of a collection of FORTRAN statements. However, a number of computational schemes have been proposed for certain restricted versions of the general problem, which has been characterized as "almost intractable" by $\mathrm{C}$. W. Gear [9].

Many researchers have attempted to extrapolate the effects of the oscillations from grid point to grid point. For certain problems in which the high frequencies are known in advance, Gautschi [8] developed linear multistep methods which are exact for trigonometric polynomials up to a certain degree, and later Snyder and Fleming [28] proposed an aliasing technique applicable to Certaine's method for solving ordinary differential equations. Multirevolution methods [10], [29] were first introduced by astronomers to calculate future satellite orbits by using some physical reference point such as a node, apogee or perigee; these ideas were further developed by Petzold [25] and Petzold and Gear [26], whose methods extrapolate the effects of the oscillations for many cycles by first calculating for one cycle near each grid point. Fatunla [7] also introduced schemes designed to follow many cycles with each time step.

Others less concerned with the details of the oscillations have proposed filtering techniques designed to eliminate entirely the effects of the fast modes. In their study of linear problems with well-separated, slowly varying large frequencies, Amdursky 
and $\mathrm{Ziv}$ [3] used left and right eigenvectors corresponding to the high frequencies to project the solution onto the manifold of smooth components. Lindberg [16] used temporal filters to remove the grid oscillations resulting from the applications of the trapezoidal rule. More recently Kreiss [14] has shown that for a large class of linear and nonlinear problems oscillations can be suppressed by a proper choice of initial conditions. And finally G. Majda [17] has demonstrated that for the linear problem time-filtered solutions have the full accuracy of the filtering method as long as the system has constant coefficients, the fast and slow scales have been separated, or the initial data have been prepared by Kreiss's technique; otherwise, the computed solutions are only first-order accurate.

Since, for many problems, the effects of the oscillations cannot be blindly suppressed or crudely approximated, a number of analytical-numerical methods have been proposed to further exploit the underlying mathematical structure. Miranker and Hoppensteadt [11], [18], [19] analyzed the theoretical and practical difficulties of implementing a method of averaging for such problems; however, they only executed their strategy to solve linear equations with constant coefficients. Amdursky and Ziv [2] also studied the linear problem with slowly varying large frequencies by using a formulation similar to averaging. Nonlinear problems of the form

$$
\begin{gathered}
d \mathbf{X} / d t=(A / \varepsilon) \mathbf{X}+\mathbf{g}(t, \mathbf{X}), \quad t>0, \\
\mathbf{X}(0)=\mathbf{X}_{0}, \quad A=\left[\begin{array}{cc}
0 & 1 \\
-1 & 0
\end{array}\right], \quad 0<\varepsilon \ll 1,
\end{gathered}
$$

were studied by Miranker and van Veldhuizen [20], who introduced a Fourier expansion in the fast scale $(\zeta=t / \varepsilon)$. Miranker and Wahba [18], [21] also analyzed such oscillations by developing a calculus of stable averaging functionals to replace the standard point functionals of analysis.

While our approach is similar to this last group in that we use analytical as well as numerical techniques to calculate the solutions accurately, we treat nonlinear systems in considerably greater generality than has previously been attempted. To illustrate the approach, we first consider the scalar problem

$$
u^{\prime}=(i a / \varepsilon) u+u^{2}, \quad u(0, \varepsilon)=u_{0}, \quad 0<t<T, 0<\varepsilon \ll 1,
$$

where $a$ is a nonzero real number and, in accordance with standard notation, $\varepsilon$ is a sufficiently small positive real number. The substitution

$$
u=\exp (\text { iat } / \varepsilon) x
$$

reduces the stiff system (1.3) to a formulation in which the right-hand side is bounded but rapidly oscillating:

$$
x^{\prime}=\exp (\text { iat } / \varepsilon) x^{2}, \quad x(0, \varepsilon)=x_{0}=u_{0}, \quad 0<t<T .
$$

In this introduction we refer to terms with factors such as $\exp ($ iat $/ \varepsilon)$ as oscillatory; terms without such factors are called nonoscillatory. For sufficiently small $\varepsilon$ system (1.5) can be solved explicitly by separation of variables

$$
\begin{aligned}
x & =x_{0}\left[1-\varepsilon x_{0}(\exp (i a t / \varepsilon)-1) /(i a)\right]^{-1} \\
& =x_{0} \sum_{k=0}^{\infty}\left[x_{0}(\exp (i a t / \varepsilon)-1) /(i a)\right]^{k} \varepsilon^{k} .
\end{aligned}
$$


For less tractable equations, or course, this method is unworkable, and solutions must be uncovered by more general techniques. On investigating the dominant balance of (1.5), we intuitively expect the rapidly oscillating terms to be less important, and accordingly in Section 4 we demonstrate that

$$
\max _{t}\left|x(t, \varepsilon)-x_{0}\right|=O(\varepsilon) .
$$

This analysis leads to an obvious change of variables:

$$
\tilde{x}^{\prime}=\exp (\text { iat } / \varepsilon)\left[\left(x_{0}^{2} / \varepsilon\right)+2 x_{0} \tilde{x}+\varepsilon \tilde{x}^{2}\right], \quad \tilde{x}(0, \varepsilon)=0, \quad x=x_{0}+\varepsilon \tilde{x} .
$$

The $O(1 / \varepsilon)$ oscillatory term cannot be neglected; however, after the substitution

$$
\tilde{x}=y_{1}(t, \varepsilon)+\hat{x}, \quad y_{1}(t, \varepsilon)=-i\left(x_{0}^{2} / a\right) \exp (\text { iat } / \varepsilon),
$$

we have the more manageable system

$$
\begin{gathered}
\hat{x}^{\prime}=\left(-2 i x_{0}^{3} / a\right) \exp (i 2 a t / \varepsilon)+2 x_{0} \hat{x} \exp (i a t / \varepsilon) \\
+\varepsilon\left[\left(-i x_{0}^{2} / a\right) \exp (i a t / \varepsilon)+\hat{x}\right]^{2} \exp (\text { iat } / \varepsilon), \\
\hat{x}(0, \varepsilon)=i\left(x_{0}^{2} / a\right),
\end{gathered}
$$

and again, by the results of Section 4 , we can neglect oscillatory terms and $O(\varepsilon)$ terms to give

$$
\max _{t}\left|\hat{x}(t, \varepsilon)-w_{1}(t)\right|=O(\varepsilon),
$$

where $w_{1}$ satisfies the system

$$
w_{1}^{\prime}=0, \quad w_{1}(0)=i\left(x_{0}^{2} / a\right) .
$$

Thus, the first-order approximation to the solution of (1.5) is given by

$$
x=x_{0}+\varepsilon\left(w_{1}(t)+y_{1}(t, \varepsilon)\right)+O\left(\varepsilon^{2}\right),
$$

where $w_{1}(t)$ is nonoscillatory and $y_{1}(t, \varepsilon)$ is oscillatory.

We systematically develop this methodology for nonlinear systems in Sections 2, 3 , and 4 , where the balancing of terms is justified by a functional Newton iteration. Integration by parts yields the first oscillatory correction as in (1.9), whereupon the elimination of secondary terms determines the first nonoscillatory correction as in (1.12). When this procedure is repeated after linearization, corrections of higher order are likewise generated; the solution is then represented by an asymptotic expansion of the form

$$
x(t, \varepsilon) \sim \sum_{k}\left(w_{k}(t)+y_{k}(t, \varepsilon)\right) \varepsilon^{k},
$$

where each $w_{k}(t)$ is bounded and nonoscillatory and each $y_{k}(t, \varepsilon)$ is bounded and oscillatory. We characterize the terms of (1.14) as the solutions of equations which are easily resolved with a large time step, that is, a time step which need not be small compared with $\varepsilon$. Given this asymptotic representation for the solution, we develop in Section 5 a formal procedure which generates the terms of the series so that repeated linearizations are unnecessary; moreover, our formalism is well-suited to computational implementation since the analytic manipulations are simply the Taylor expansions of polynomials. Our approach is conceptually similar to the generalized method of averaging as developed by Bogoliubov and Mitropolsky [4]. 
One can treat problems with variable coefficients in a similar fashion. For example, we consider a nonautonomous variant of (1.5),

$$
x^{\prime}=\exp (i a(t) / \varepsilon) x^{2}, \quad x(0, \varepsilon)=x_{0}, \quad 0<t<T,
$$

where $a(t)$ is a smooth real function with

$$
\min _{t}\left|a^{\prime}(t)\right|>0 \text {. }
$$

As in (1.6) the solution is readily obtained by separation of variables:

$$
x=x_{0}\left[1-x_{0} F(t, \varepsilon)\right]^{-1}=x_{0} \sum_{k=0}^{\infty}\left[x_{0} F(t, \varepsilon)\right]^{k},
$$

where

$$
F(t, \varepsilon)=\int_{0}^{t} \exp (i a(t) / \varepsilon) d t .
$$

The right-hand side of (1.18) can be integrated by parts to give an asymptotic expansion

$$
\left.F(t, \varepsilon) \sim \exp (i a(t) / \varepsilon)\left\{\varepsilon\left[-i / a^{\prime}(t)\right]+\varepsilon^{2}\left[a^{\prime \prime}(t) /\left(a^{\prime}(t)\right)^{3}\right]+O\left(\varepsilon^{3}\right)\right\}\right|_{0} ^{t},
$$

and after the substitution of (1.19) into (1.17) we have an expansion of the form given in (1.14). Without the assumption (1.16) this procedure is unworkable because the mathematical structure of $F(t, \varepsilon)$ changes significantly over any interval where $a^{\prime}(t)$ vanishes. This behavior characterizes the general theory, where the expansions first may become nonuniform and then eventually break down entirely due to the failure of integration by parts.

In a future paper we shall extend our results so as to handle these circumstances, which can be described mathematically as a turning point or physically as a passage through resonance. In general, however, if there is no well-defined separation between the frequencies of the fast and slow modes of a system, then one is not really solving a problem with different time scales.

2. Reduction to the Nonstiff Formulation. We consider the general system

$$
\mathbf{Z}^{\prime}=(A(t) / \varepsilon) \mathbf{Z}+\mathbf{H}(\mathbf{Z}, t), \quad \mathbf{Z}(0, \varepsilon)=\hat{\mathbf{Z}}_{0}, \quad 0<t<T, 0<\varepsilon \ll 1,
$$
where:

(i) $\mathbf{Z}$ is an $n$-dimensional complex vector.

(ii) $\hat{\mathbf{Z}}_{0}$ is independent of $\varepsilon$.

(iii) $\mathbf{H}(\mathbf{Z}, t)$ has components which are polynomial in the components of $\mathbf{Z}$ with $t$-dependent coefficients in $C^{p}(t)(p>0)$.

(iv) The matrix $A(t)$ is in diagonal form with purely imaginary entries:

$$
A(t)=\operatorname{diag}_{k}\left(\lambda_{k}(t)\right) \quad\left(\operatorname{Re}\left\{\lambda_{k}(t)\right\}=0\right), \quad \Lambda(t)^{(k)}=\lambda_{k}(t) \in C^{p}(t) .
$$

Here $\Lambda(t)^{(k)}$ is the $k$ th component of the vector $\Lambda(t)$, and $C^{p}(t)$ is the set of all $t$-dependent functions with $p$ continuous derivatives. In this paper $|\mathbf{f}|$ denotes the maximum norm of the vector $f$. If the nonlinearities are not polynomial, then one often can make local approximations to achieve this form. In any case smoothness requirements appear to be necessary for both the independent and the dependent variables. The assumption on the diagonal structure of $A(t)$ is needed to guarantee a 
bound on the growth of the solution. For example, the matrix

$$
\frac{1}{\varepsilon}\left[\begin{array}{ll}
i & 1 \\
0 & i
\end{array}\right]+\left[\begin{array}{ll}
0 & 0 \\
d & 0
\end{array}\right]
$$

has eigenvalues

$$
\lambda .=i / \varepsilon \pm \sqrt{d / \varepsilon} .
$$

Thus, if $A(t)$ has such Jordan structure, then an $O(1)$ perturbation of the system will cause the solution to become unbounded as

$$
\varepsilon \rightarrow 0 \text {. }
$$

We also note that a smooth $\varepsilon$-dependency in the coefficients and the initial conditions is possible although this adds no significant features to the theory.

This system can be transformed to a formulation in which the coefficients are bounded but some are rapidly oscillating.

Example 1. A simple mass-spring system with small damping can be modeled by the equation for a Rayleigh oscillator:

$$
\begin{gathered}
\left(\begin{array}{l}
z_{1} \\
z_{2}
\end{array}\right)^{\prime}=\left[\begin{array}{cc}
0 & 1 / \varepsilon \\
-1 / \varepsilon & 0
\end{array}\right]\left(\begin{array}{l}
z_{1} \\
z_{2}
\end{array}\right)+\left(\begin{array}{c}
0 \\
z_{2}-\left(z_{2}\right)^{3} / 3
\end{array}\right), \\
\left(\begin{array}{l}
z_{1}(0, \varepsilon) \\
z_{2}(0, \varepsilon)
\end{array}\right)=\left(\begin{array}{l}
1 \\
0
\end{array}\right), \quad 0<t<T, 0<\varepsilon \ll 1 .
\end{gathered}
$$

After the change of variables

$$
\begin{gathered}
\left(\begin{array}{l}
z_{1} \\
z_{2}
\end{array}\right)=S\left(\begin{array}{l}
u_{1} \\
u_{2}
\end{array}\right)=\frac{1}{2}\left[\begin{array}{cc}
1 & 1 \\
-i & i
\end{array}\right]\left(\begin{array}{l}
u_{1} \\
u_{2}
\end{array}\right)=\left(\begin{array}{c}
\left(u_{1}+u_{2}\right) / 2 \\
i\left(u_{1}-u_{2}\right) / 2
\end{array}\right), \\
\left(\begin{array}{l}
u_{1} \\
u_{2}
\end{array}\right)=S^{-1}\left(\begin{array}{l}
z_{1} \\
z_{2}
\end{array}\right)=\left[\begin{array}{cc}
1 & i \\
1 & -i
\end{array}\right]\left(\begin{array}{l}
z_{1} \\
z_{2}
\end{array}\right)=\left(\begin{array}{l}
z_{1}+i z_{2} \\
z_{1}-i z_{2}
\end{array}\right),
\end{gathered}
$$

the equations become

$$
\begin{gathered}
\left(\begin{array}{l}
u_{1} \\
u_{2}
\end{array}\right)^{\prime}=\left[\begin{array}{cc}
-i / \varepsilon & 0 \\
0 & i / \varepsilon
\end{array}\right]\left(\begin{array}{l}
u_{1} \\
u_{2}
\end{array}\right)+\left(\begin{array}{c}
F\left(u_{1}, u_{2}\right) \\
-F\left(u_{1}, u_{2}\right)
\end{array}\right), \\
\left(\begin{array}{l}
u_{1}(0, \varepsilon) \\
u_{2}(0, \varepsilon)
\end{array}\right)=\left(\begin{array}{l}
1 \\
1
\end{array}\right), \quad 0<t<T,
\end{gathered}
$$

where

2.9) $F\left(u_{1}, u_{2}\right)=\frac{1}{2} u_{1}-\frac{1}{2} u_{2}-\frac{1}{24} u_{2}^{3}+\frac{1}{8} u_{2}^{2} u_{1}-\frac{1}{8} u_{2} u_{1}^{2}+\frac{1}{24} u_{1}^{3}, \quad u_{2}=\bar{u}_{1}$.

The system (2.8) has the form (2.1). Next we make the change of variables

$$
u_{1}=\exp (-i t / \varepsilon) x_{1}, \quad u_{2}=\exp (i t / \varepsilon) x_{2},
$$

and obtain

$$
\left(\begin{array}{l}
x_{1} \\
x_{2}
\end{array}\right)^{\prime}=\left(\begin{array}{c}
\exp (i t / \varepsilon) F\left(\exp (-i t / \varepsilon) x_{1}, \exp (i t / \varepsilon) x_{2}\right) \\
-\exp (-i t / \varepsilon) F\left(\exp (-i t / \varepsilon) x_{1}, \exp (i t / \varepsilon) x_{2}\right)
\end{array}\right)
$$


by which we have

$$
\begin{aligned}
x_{1}^{\prime}= & \frac{1}{2} x_{1}-\frac{1}{8} x_{2} x_{1}^{2}-\frac{1}{2} \exp (2 i t / \varepsilon) x_{2}-\frac{1}{24} \exp (4 i t / \varepsilon) x_{2}^{3} \\
+ & \frac{1}{8} \exp (2 i t / \varepsilon) x_{2}^{2} x_{1}+\frac{1}{24} \exp (-2 i t / \varepsilon) x_{1}^{3} \\
& x(0, \varepsilon)=1, \quad x_{2}=\bar{x}_{1}, \quad 0<t<T .
\end{aligned}
$$

For the general case, let $S_{\varepsilon}(t, s)$ be the solution operator of the reduced system (2.1); in fact, we can write

$$
S_{\varepsilon}(t, s)=\underset{k}{\operatorname{diag}}\left\{\exp \left[\left(\int_{s}^{t} \lambda_{k}(\tau) d \tau\right) / \varepsilon\right]\right\} .
$$

Thus, with the change of variables

$$
\mathbf{X}=S_{\varepsilon}(t, 0)^{-1} \mathbf{Z},
$$

we reach a system in which the coefficients are bounded but some are rapidly oscillating:

$$
\begin{gathered}
\mathbf{X}^{\prime}=\mathbf{G}(\mathbf{X}, t, \varepsilon)=\mathbf{g}_{\mathrm{I}}(\mathbf{X}, t, \varepsilon)+\mathbf{g}_{\mathrm{II}}(\mathbf{X}, t)+\mathbf{f}_{\mathrm{I}}(t, \varepsilon)+\mathbf{f}_{\mathrm{II}}(t), \\
\mathbf{X}(0, \varepsilon)=\hat{\mathbf{X}}_{0} ; \quad 0<\varepsilon \ll 1 ; 0<t<T .
\end{gathered}
$$

Here the forms of the coefficients are given by

(i) $\hat{\mathbf{X}}_{0}=\hat{\mathbf{Z}}_{0}$ is independent of $\varepsilon$.

(ii) $g_{\mathrm{I}}(\mathbf{X}, t, \varepsilon)^{(i)}=\sum_{j} a_{i j}(t) \exp \left[B_{i j}(t) / \varepsilon\right] p_{i j}(\mathbf{X})$.

(iii) $g_{\mathrm{II}}(\mathbf{X}, t)^{(i)}=\sum_{j} d_{i j}(t) q_{i j}(\mathbf{X})$.

(iv) $f_{\mathrm{I}}(t, \varepsilon)^{(i)}=\sum_{j} c_{i j}(t) \exp \left[G_{i j}(t) / \varepsilon\right]$.

(v) $f_{\mathrm{II}}(t)^{(i)}=h_{i}(t)$.

(vi) $\left\{a_{i j}(t), d_{i j}(t), c_{i j}(t), h_{i}(t)\right\} \subset C^{p}(t)$.

$p_{i j}(\mathbf{X})$ and $q_{i j}(\mathbf{X})$ are monomials of positive degree in the components of $\mathbf{X} ; B_{i j}(t) / \varepsilon$ and $G_{i j}(t) / \varepsilon$ can be represented by $n$-vector scalar products of the form

$$
\mathbf{N}^{T} \mathbf{P}(t) / \varepsilon
$$

where $\mathbf{N}$ is a constant $n$-vector with integral components and the elements of $\mathbf{P}(t)$ are given by

$$
P(t)^{(i)}=\int_{0}^{t} \lambda_{i}(s) d s .
$$

By (2.2) and (2.17) the $t$-derivative of the expression (2.16) is

$$
\left(\mathbf{N}^{T} \mathbf{P}(t) / \varepsilon\right)^{\prime}=\mathbf{N}^{T} \boldsymbol{\Lambda}(t) / \varepsilon
$$

The entries of $\Lambda(t) / \varepsilon$ are called fundamental frequencies, while the relevant terms of the form (2.18) are called secondary frequencies. We also impose the following restriction on all relevant secondary frequencies:

$$
\left|\mathbf{N}^{T} \boldsymbol{\Lambda}(t)\right|>K>0,
$$

where $K$ is some positive constant. $K / \varepsilon$ is then a measure of the stiffness of the system.

This last assumption arises out of the necessity for some concrete specification as to the meaning of "fast oscillations"; moreover, this restriction must be maintained in subsequent levels of analysis. Functions which have the form given by (iv) and 
which satisfy (2.19) are called strictly oscillatory (of class $p$ ); functions of the form given by ( $\mathrm{v}$ ) are called strictly nonoscillatory ( of class $p$ ). In this paper the subscript I designates a strictly oscillatory function, and the subscript II designates a strictly nonoscillatory function. The system (2.15) is said to be in nonstiff oscillatory form.

If for some relevant

$$
\sigma(t)=\mathbf{N}^{T} \mathbf{P}(t)
$$

we have

$$
\sigma^{\prime}(t)=\mathbf{N}^{T} \boldsymbol{\Lambda}(t) \equiv 0 .
$$

then the corresponding term can be reclassified as nonoscillatory; however, if $\sigma^{\prime}(t)$ vanishes at some isolated point, then the approximation must be made as a turning-point calculation, and, as previously noted, this procedure will be outlined in another paper. If, as in Example 1, the entries of $\Lambda(t)$ are integral constants, this difficulty cannot occur since all possible secondary frequencies must satisfy (2.19) or (2.21) with

$$
K=1 \text {. }
$$

The strength of the restriction (2.19) also allows us to define the leading-order antiderivative of any oscillatory function through the linear operator

$$
\mathcal{L}\{\mathbf{c}(t) \exp [B(t) / \varepsilon]\}=\left[\mathbf{c}(t) / B^{\prime}(t)\right] \exp [B(t) / \varepsilon],
$$

since

$$
\varepsilon \mathcal{L}\{\mathbf{c}(t) \exp [B(t) / \varepsilon]\}^{\prime}=\mathbf{c}(t) \exp [B(t) / \varepsilon]+O(\varepsilon),
$$

where the $O(\varepsilon)$ notation is to be interpretated in terms of the maximum norm.

Our aim is to characterize the solution of the system (2.15) in terms of these concepts. Thus, the function $\mathbf{f}(t, \varepsilon)$ is said to be decomposable if it is of the form

$$
\mathbf{f}(t, \varepsilon)=\sum_{k}\left(\mathbf{W}_{k}(t)+\mathbf{Y}_{k}(t, \varepsilon)\right) \varepsilon^{k}
$$

where each $\mathbf{Y}_{k}(t, \varepsilon)$ is strictly oscillatory and each $\mathbf{W}_{k}(t)$ is strictly nonoscillatory. And likewise $f(t, \varepsilon)$ is said to be decomposable to $O\left(\varepsilon^{m}\right)$ if

$$
\mathbf{f}(t, \varepsilon)=\sum_{k}\left(\mathbf{W}_{k}(t)+\mathbf{Y}_{k}(t, \varepsilon)\right) \varepsilon^{k}+O\left(\varepsilon^{m+1}\right),
$$

where each $\mathbf{Y}_{k}(t, \varepsilon)$ is strictly oscillatory and each $\mathbf{W}_{k}(t)$ is strictly nonoscillatory. The characterization of the solution of (2.15) in terms of such an asymptotic expansion stands as the major goal of this paper; the breakdown of this decomposability principle corresponds to a violation of (2.19), whereupon turning-point techniques are necessary.

3. Hierarchy for the Linear Problem. Since our treatment is based on a functional Newton iteration, we first discuss the linear problem

$$
\begin{gathered}
\mathbf{X}^{\prime}=A_{\mathrm{I}}(t, \varepsilon) \mathbf{X}+A_{\mathrm{II}}(t) \mathbf{X}+\mathbf{f}_{\mathrm{I}}(t, \varepsilon)+\mathbf{f}_{\mathrm{II}}(t), \\
\mathbf{X}(0, \varepsilon)=\hat{\mathbf{X}}_{0} ; \quad 0<t<T, 0<\varepsilon \ll 1,
\end{gathered}
$$

where the subscript I denotes a strictly oscillatory function and the subscript II denotes a strictly nonoscillatory function. We begin with a rather standard result on the stability of ordinary differential equations. 
LEMMA [3.1]. Consider the two systems

$$
\mathbf{Y}^{\prime}=B(t) \mathbf{Y}+\mathbf{h}(t, \varepsilon), \quad \mathbf{Y}(0, \varepsilon)=Y_{0}, \quad 0<t<T, 0<\varepsilon \ll 1,
$$

and

$$
\begin{gathered}
\mathbf{Z}^{\prime}=B(t) \mathbf{Z}+\mathbf{h}(t, \varepsilon)+\varepsilon \tilde{\mathbf{h}}(t, \varepsilon), \\
\mathbf{Z}(0, \varepsilon)=\mathbf{Y}_{0}, \quad 0<t<T, 0<\varepsilon \ll 1,
\end{gathered}
$$

where each vector or matrix is a bounded continuous function of its arguments. Then we have

$$
\max _{t}|\mathbf{Y}(t, \varepsilon)-\mathbf{Z}(t, \varepsilon)|=O(\varepsilon), \quad \max _{t}\left|\mathbf{Y}^{\prime}(t, \varepsilon)-\mathbf{Z}^{\prime}(t, \varepsilon)\right|=O(\varepsilon)
$$

Proof. The system for

$$
\mathbf{R}=\mathbf{Y}-\mathbf{Z}
$$

has the form

$$
\mathbf{R}^{\prime}=B(t) \mathbf{R}-\varepsilon \tilde{\mathbf{h}}(t, \varepsilon), \quad \mathbf{R}(0, \varepsilon)=0,
$$

and therefore by the basic results of stability theory we have (3.4) (see, for example, Coddington and Levinson [6]).

Thus, to achieve leading-order accuracy one simply ignores certain terms of the system. This principle leads to the following useful result concerning the system (3.1).

THEOREM [3.1]. Let $\mathbf{X}(t, \varepsilon)$ be the solution of (3.1), and let $\mathbf{V}(t)$ be the solution of the system

$$
\mathbf{V}^{\prime}=A_{\mathrm{II}}(t) \mathbf{V}+\mathbf{f}_{\mathrm{II}}(t), \quad \mathbf{V}(0)=\hat{\mathbf{X}}_{0}
$$

We then have

$$
\begin{gathered}
\max _{t}|\mathbf{X}(t, \varepsilon)-\mathbf{V}(t)|=O(\varepsilon), \quad \max _{t}\left|\mathbf{X}^{\prime}(t, \varepsilon)-\mathbf{V}^{\prime}(t)-\varepsilon F^{\prime}(t, \varepsilon)\right|=O(\varepsilon), \\
\mathbf{F}(t, \varepsilon)=\mathcal{E}\left\{\mathbf{f}_{\mathrm{I}}+A_{\mathrm{I}} \mathbf{V}\right\} .
\end{gathered}
$$

Proof. Let

$$
\mathbf{Z}(t, \varepsilon)=\mathbf{X}(t, \varepsilon)-\mathbf{V}(t)
$$

Then the equations for $\mathbf{Z}(t, \varepsilon)$ are

$$
\mathbf{Z}^{\prime}=\left(A_{\mathrm{I}}(t, \varepsilon)+A_{\mathrm{II}}(t)\right) \mathbf{Z}+\left(\mathbf{f}_{\mathrm{I}}(t, \varepsilon)+A_{\mathrm{I}}(t, \varepsilon) \mathbf{V}(t)\right), \quad \mathbf{Z}(0, \varepsilon)=0 .
$$

Since $\mathbf{V}(t)$ is strictly nonoscillatory, both forcing terms are strictly oscillatory. Then by (2.24) the equations for

$$
\tilde{\mathbf{Z}}=\mathbf{Z}-\varepsilon(\mathbf{F}(t, \varepsilon)-\mathbf{F}(0, \varepsilon))
$$

have the form

$$
\tilde{\mathbf{Z}}^{\prime}=\left(A_{\mathrm{I}}(t, \varepsilon)+A_{\mathrm{II}}(t)\right) \tilde{\mathbf{Z}}+\varepsilon \mathbf{h}(t, \varepsilon), \quad \tilde{\mathbf{Z}}(0, \varepsilon)=0,
$$

and so by Lemma [3.1]:

$$
\max _{t}|\mathbf{Z}(t, \varepsilon)|=O(\varepsilon), \quad \max _{t}\left|\mathbf{Z}^{\prime}(t, \varepsilon)-\varepsilon \mathbf{F}^{\prime}(t, \varepsilon)\right|=O(\varepsilon) .
$$


4. Solution by Successive Linearizations. By using the results of the previous section, we now generate an asymptotic expansion for the solution of the system (2.15), which is in nonstiff oscillatory form. If a linearization technique is to be successful, one must have a suitable value for the initial approximation.

Assumption [4a]. In correspondence to the system (2.15), the reduced system

$$
\mathbf{V}^{\prime}=\mathbf{g}_{\mathrm{II}}(\mathbf{V}, t)+\mathbf{f}_{\mathrm{II}}(t), \quad \mathbf{V}(0)=\hat{\mathbf{X}}_{0}, \quad 0<t<T .
$$

is well-posed and has a bounded solution in $C^{p+1}(t)$.

Given this assumption, we can define the $(n \times n)$ matrices

$$
A_{\mathrm{I}}(t, \varepsilon)=\left.\mathbf{g}_{\mathrm{I}}(\mathbf{X}, t, \varepsilon)_{\mathbf{X}}\right|_{\mathbf{X}=\mathbf{v}}, \quad A_{\mathrm{II}}(t)=\left.\mathbf{g}_{\mathrm{II}}(\mathbf{X}, t)_{\mathbf{X}}\right|_{\mathbf{X}=\mathbf{v}},
$$

where $A_{\mathrm{I}}(t, \varepsilon)$ is strictly oscillatory of class $p$ and $A_{\mathrm{II}}(t)$ is strictly nonoscillatory of class $p$. Here the notation $\mathbf{g}(\mathbf{X})_{\mathbf{X}}$ indicates the Jacobian of the vector function. We also define the operator

$$
\because(\mathbf{X})=\mathbf{G}(\mathbf{X}, t, \varepsilon)-\mathbf{X}^{\prime}
$$

where $\mathbf{G}(\mathbf{X}, t, \varepsilon)$ is as given in (2.15). For a positive integer $m, \tilde{\mathbf{X}}^{m}$ is said to be an $\varepsilon^{m}$-approximate solution of $(2.15)$ if

$$
\begin{aligned}
\tilde{\mathbf{X}}^{m}(t, \varepsilon) & =\sum_{k=0}^{m} \varepsilon^{k}\left(\mathbf{W}_{k}(t)+\mathbf{Y}_{k}(t, \varepsilon)\right)+\varepsilon^{m+1} \mathbf{Y}_{m+1}(t, \varepsilon), \\
\tilde{\mathbf{X}}^{m}(0, \varepsilon) & =\hat{\mathbf{X}}_{0}+O\left(\varepsilon^{m+1}\right), \quad \text { 代 }\left(\tilde{\mathbf{X}}^{m}\right)=O\left(\varepsilon^{m+1}\right),
\end{aligned}
$$

where each $\mathbf{Y}_{k}$ is strictly oscillatory and each $\mathbf{W}_{k}$ is strictly nonoscillatory. By means of Assumption [4a] we immediately can demonstrate the existence of such an approximate solution.

THEOREM [4.1] The function $\tilde{\mathbf{X}}^{0}$, which is given by

$$
\begin{gathered}
\tilde{\mathbf{X}}^{0}(t, \tau)=\mathbf{W}_{0}(t)+\varepsilon \mathbf{Y}_{1}(t, \varepsilon), \quad \mathbf{W}_{0}(t)=\mathbf{V}(t), \\
\mathbf{Y}_{1}(t, \varepsilon)=\mathcal{L}\left\{\mathbf{g}_{\mathrm{I}}(\mathbf{V}, t, \varepsilon)+\mathbf{f}_{\mathrm{I}}(t, \varepsilon)\right\},
\end{gathered}
$$

is an $\varepsilon^{0}$-approximatate solution of the system $(2.15) . \mathbf{W}_{0}(t)$ is strictly nonoscillatory of class $(p+1)$, and $\mathbf{Y}_{1}(t, \varepsilon)$ is strictly oscillatory of class $p$.

Proof. To verify (4.4) we consider

$$
\text { OK }\left(\tilde{\mathbf{X}}^{0}\right)=\mathbf{G}\left(\tilde{\mathbf{X}}^{0}, t, \varepsilon\right)-\left(\tilde{\mathbf{X}}^{0}\right)^{\prime} .
$$

By (4.1) and (2.24) we have

$$
\left(\tilde{\mathbf{X}}^{0}\right)^{\prime}=\mathbf{V}^{\prime}+\mathbf{g}_{\mathrm{I}}(\mathbf{V}, t, \varepsilon)+\mathbf{f}_{\mathrm{I}}(t, \varepsilon)+O(\varepsilon)=\mathbf{G}(\mathbf{V}, t, \varepsilon)+O(\varepsilon),
$$

and also a simple Taylor expansion gives

$$
\mathbf{G}\left(\tilde{\mathbf{X}}^{0}, t, \varepsilon\right)=\mathbf{G}(\mathbf{V}, t, \varepsilon)+O(\varepsilon) .
$$

Therefore $\tilde{\mathbf{X}}^{0}$ is an $\varepsilon^{0}$-approximate solution of the system (2.15).

We now demonstrate that an $\varepsilon^{m}$-approximate solution actually approximates the exact solution of the system.

THEOREM [4.2]. If $\tilde{\mathbf{X}}^{m}$ is an $\varepsilon^{m}$-approximate solution of the system (2.15), then

$$
\max _{t}\left|\tilde{\mathbf{X}}^{m}(t, \varepsilon)-\mathbf{X}(t, \varepsilon)\right|=O\left(\varepsilon^{m+1}\right),
$$

where $\mathbf{X}(t, \varepsilon)$ is the solution of $(2.15)$. 
Proof. Consider the $(n+1)$-dimensional space

$$
\text { 미 }=\left\{(\mathbf{x}, t) \ni\left|\mathbf{x}-\tilde{\mathbf{X}}^{m}\right|<\delta, 0<t<T\right\},
$$

where $\delta$ is some arbitrary positive constant. By our assumptions on $\tilde{\mathbf{X}}^{m}$ and the system (2.15), we conclude that for some positive constants $K_{1}$ and $K_{2}$ we have:

(i) $\mathbf{G}(\mathbf{x}, t, \varepsilon)$ is continuous in $2 / 2$;

(ii) $|\mathbf{G}(\mathbf{x}, t, \varepsilon)|<K_{1}$ in o 2 ;

(iii) $\left|\mathbf{G}\left(\mathbf{x}_{1}, t, \varepsilon\right)-\mathbf{G}\left(\mathbf{x}_{2}, t, \varepsilon\right)\right|<K_{2}\left|\mathbf{x}_{1}-\mathbf{x}_{2}\right|$ in ( ) $)$.

Note that the Lipschitz condition (iii) is guaranteed even though the $t$-derivatives of $\mathbf{G}(\mathbf{x}, t, \varepsilon)$ are unbounded as $\varepsilon \rightarrow 0$. We now introduce a sequence of Picard iterates:

$$
\mathbf{x}_{0}=\tilde{\mathbf{X}}^{m}, \quad \mathbf{x}_{N+1}=\hat{\mathbf{X}}_{0}+\int_{0}^{t} \mathbf{G}\left(\mathbf{x}_{N}, t, \varepsilon\right) d t .
$$

Since $\tilde{\mathbf{X}}^{m}$ satisfies the equation to within $O\left(\varepsilon^{m+1}\right)$ we have

$$
\left|\mathbf{x}_{1}-\mathbf{x}_{0}\right|=\left|\tilde{\mathbf{X}}^{m}-\hat{\mathbf{X}}_{0}-\int_{0}^{t} \mathbf{G}\left(\tilde{\mathbf{X}}^{m}, t, \varepsilon\right) d t\right|<R \varepsilon^{m+1},
$$

where $R$ is a positive constant. Provided the successive iterates are all in $(2)$, we have by induction

$$
\left|\mathbf{x}_{N+1}-\mathbf{x}_{N}\right|<\varepsilon^{m+1} R\left(K_{2} t\right)^{N} / N !,
$$

and thus for all positive $N$

$$
\max _{t}\left|\mathbf{x}_{N}-\mathbf{x}_{0}\right|<\varepsilon^{m+1} R \exp \left(K_{2} T\right) .
$$

Therefore, for sufficiently small $\varepsilon$ all iterates remain in 12 . By the uniform convergence of the iteration, we have the existence of a unique continuously differentiable function $\mathbf{X}$ which satisfies

$$
\because(\mathbf{X})=0, \quad \mathbf{X}(0, \varepsilon)=\hat{\mathbf{X}}_{0}, \quad \max _{t}\left|\tilde{\mathbf{X}}^{m}-\mathbf{X}\right|=O\left(\varepsilon^{m+1}\right) .
$$

COROllary [4.2]. The system (2.15) with Assumption [4a] is well-posed with

$$
\max _{t}|\mathbf{V}(t)-\mathbf{X}(t, \varepsilon)|=O(\varepsilon),
$$

where $\mathbf{X}(t, \varepsilon)$ is the solution of $(2.15)$.

Proof. Since an $\varepsilon^{0}$-approximate solution is given by Theorem [4.1], the error estimate follows from Theorem [4.2].

By using Theorem [4.2] we now extend the result of Corollary [4.2] to obtain higher-order approximations.

THEOREM [4.3]. Consider the sistem (2.15). Let $\tilde{\mathbf{X}}^{m}$ be an $\varepsilon^{m}$-approximate solution where, for $k>1, \mathbf{W}_{k}$ is strictly nonoscillatory of class $(p+2-k)$ and $\mathbf{Y}_{k}$ is strictly oscillatory of class $(p+1-k)$. Let $)\left(\tilde{\mathbf{X}}^{m}\right)$ be decomposable to $O\left(\varepsilon^{m+1}\right)$ with the form

$$
\mathcal{O}\left(\tilde{\mathbf{X}}^{m}\right)=\varepsilon^{m+1} \mathbf{f}_{\mathrm{I}}+\varepsilon^{m+1} \mathbf{f}_{\mathrm{II}}+O\left(\varepsilon^{m+2}\right),
$$

where $\mathbf{f}_{\mathrm{I}}$ is strictly oscillatory of class $(p-m-1)$ and $\mathbf{f}_{\mathrm{II}}$ is strictly nonoscillatory of class $(p-m)$. Then an $\varepsilon^{m+1}$-approximate solution of the system is given by

$$
\tilde{\mathbf{X}}^{m+1}=\tilde{\mathbf{X}}^{m}+\varepsilon^{m+1} \mathbf{W}_{m+1}+\varepsilon^{m+2} \mathbf{Y}_{m+2},
$$


where

$$
\begin{gathered}
\mathbf{W}_{m+1}^{\prime}=A_{\mathrm{II}}(t) \mathbf{W}_{m+1}+\mathbf{f}_{\mathrm{II}}(t), \quad \mathbf{W}_{m+1}(0)=-\mathbf{Y}_{m+1}(0, \varepsilon), \\
\mathbf{Y}_{m+2}(t, \varepsilon)=\mathcal{E}\left\{\mathbf{f}_{\mathrm{I}}+A_{\mathrm{I}} \mathbf{W}_{m+1}\right\} .
\end{gathered}
$$

Here $\mathbf{W}_{m+1}$ is strictly nonoscillatory of class $(p-m-1)$, and $\mathbf{Y}_{m+2}$ is strictly oscillatory of class $(p-m-1)$. Thus, we have

$$
\left|\mathbf{X}-\tilde{\mathbf{X}}^{m+1}\right|=O\left(\varepsilon^{m+2}\right), \quad \tilde{\mathbf{X}}^{m+1}=\sum_{k=0}^{m+1} \varepsilon^{k}\left(\mathbf{W}_{k}+\mathbf{Y}_{k}\right)+\varepsilon^{m+2} \mathbf{Y}_{m+2}
$$

Moreover, OM( $\left(\tilde{\mathbf{X}}^{m+1}\right)$ has the form $(4.8)$ with $m$ replaced by $(m+1)$ if

(1) $\mathfrak{R}\left(\tilde{\mathbf{X}}^{m}\right)$ is decomposable to $O\left(\varepsilon^{m+2}\right)$;

(2) $A_{\mathrm{I}}(t, \varepsilon) \mathbf{Y}_{m+2}$ is decomposable; and

(3) $\left.\left[\left(\mathbf{G}(\mathbf{X}, t, \varepsilon)_{\mathbf{X}}\right)\left(\mathbf{W}_{m+1}\right)\right]_{\mathbf{X}}\left(\mathbf{W}_{1}+\mathbf{Y}_{1}\right)\right|_{\mathbf{X}=\mathbf{V}}$ is decomposable.

Proof. By Theorem [4.2] we have

$$
\mathbf{X}=\tilde{\mathbf{X}}^{m}+\varepsilon^{m+1} \mathbf{Z}, \quad \mathbf{Z}(0, \varepsilon)=-\mathbf{Y}_{m+1}(0, \varepsilon),
$$

where $\mathbf{Z}(t, \varepsilon)$ is a continuously differentiable function of $t$, and $\mathbf{X}$ is the solution of (2.15). The differential equation then can be written as

$$
\left(\tilde{\mathbf{X}}^{m}+\varepsilon^{m+1} \mathbf{Z}\right)^{\prime}=\mathbf{G}\left(\tilde{\mathbf{X}}^{m}+\varepsilon^{m+1} \mathbf{Z}\right)=\mathbf{G}\left(\tilde{\mathbf{X}}^{m}+\varepsilon^{m+1} \mathbf{Z}\right)-\mathbf{G}\left(\tilde{\mathbf{X}}^{m}\right)+\mathbf{G}\left(\tilde{\mathbf{X}}^{m}\right) .
$$

We now carry out a linearization which is equivalent to a functional Newton iteration:

$$
\begin{aligned}
\mathbf{Z}^{\prime} & =\left[\mathbf{G}\left(\tilde{\mathbf{X}}^{m}+\varepsilon^{m+1} \mathbf{Z}\right)-\mathbf{G}\left(\tilde{\mathbf{X}}^{m}\right)\right] / \varepsilon^{m+1}+\mathfrak{N}\left(\tilde{\mathbf{X}}^{m}\right) / \varepsilon^{m+1} \\
& =\left[A_{\mathrm{I}}(t, \varepsilon)+A_{\mathrm{II}}(t)\right] \mathbf{Z}+\mathbf{f}_{\mathrm{I}}(t, \varepsilon)+\mathbf{f}_{\mathrm{II}}(t)+O(\varepsilon),
\end{aligned}
$$

where $A_{\text {I }}$ and $A_{\text {II }}$ are given by (4.2). By Lemma [3.1] and Theorem [3.1] we have

$$
\max _{t}\left|\mathbf{Z}-\left(\mathbf{W}_{m+1}+\varepsilon \mathbf{Y}_{m+2}\right)\right|=O(\varepsilon), \quad \max _{t}\left|\mathbf{Z}^{\prime}-\left(\mathbf{W}_{m+1}+\varepsilon \mathbf{Y}_{m+2}\right)^{\prime}\right|=O(\varepsilon),
$$

where

$$
\begin{gathered}
\mathbf{W}_{m+1}^{\prime}=A_{\mathrm{II}}(t) \mathbf{W}_{m+1}+\mathbf{f}_{\mathrm{II}}(t), \quad \mathbf{W}_{m+1}(0)=-\mathbf{Y}_{m+1}(0, \varepsilon), \\
\mathbf{Y}_{m+2}(t, \varepsilon)=\varrho\left\{\mathbf{f}_{\mathrm{I}}+A_{\mathrm{I}} \mathbf{W}_{m+1}\right\} .
\end{gathered}
$$

Since $S_{f}(0,0)=I$, by $(2.13)$, the initial condition for $\mathbf{W}_{m+1}$ is actually independent of $\varepsilon$. By our construction $\tilde{\mathbf{X}}^{m+1}$ and $\left(\tilde{\mathbf{X}}^{m+1}\right)^{\prime}$ approximate $\mathbf{X}$ and $\mathbf{X}^{\prime}$, respectively, to within $O\left(\varepsilon^{m+2}\right)$. Thus, we have

$$
\begin{aligned}
\pi\left(\tilde{\mathbf{X}}^{m+1}\right) & =\pi\left(\tilde{\mathbf{X}}^{m+1}\right)-\Re(\mathbf{X}) \\
& =\left[\mathbf{G}\left(\tilde{\mathbf{X}}^{m+1}, t, \varepsilon\right)-\mathbf{G}(\mathbf{X}, t, \varepsilon)\right]+\left[\left(\tilde{\mathbf{X}}^{m+1}\right)^{\prime}-\mathbf{X}^{\prime}\right]=O\left(\varepsilon^{m+2}\right) .
\end{aligned}
$$

Since

$$
\tilde{\mathbf{X}}^{m+1}(0, \varepsilon)=\hat{\mathbf{X}}_{0}+\varepsilon^{m+2} \mathbf{Y}_{m+2}(0, \varepsilon)
$$


we conclude that $\tilde{\mathbf{X}}^{m+1}$ is an $\varepsilon^{m+1}$-approximate solution of the system (2.15); (4.11) then follows from Theorem [4.2]. We have

$$
\begin{aligned}
& \mathscr{N}\left(\tilde{\mathbf{X}}^{m+1}\right)=\mathfrak{N}\left(\tilde{\mathbf{X}}^{m}\right)+\mathscr{N}\left(\tilde{\mathbf{X}}^{m+1}\right)-\mathfrak{N}\left(\tilde{\mathbf{X}}^{m}\right) \\
&= \Re\left(\tilde{\mathbf{X}}^{m}\right)-\varepsilon^{m+1} \mathbf{W}_{m+1}^{\prime}(t)-\varepsilon^{m+2} \mathbf{Y}_{m+2}^{\prime}(t, \varepsilon)+\varepsilon^{m+1}\left[\mathbf{G}\left(\tilde{\mathbf{X}}^{m}, t, \varepsilon\right)_{\mathbf{X}}\right] \mathbf{W}_{m+1}(t) \\
&+\varepsilon^{m+2}\left[\mathbf{G}\left(\tilde{\mathbf{X}}^{m}, t, \varepsilon\right)_{\mathbf{X}}\right] \mathbf{Y}_{m+2}(t, \varepsilon)+O\left(\varepsilon^{2 m+2}\right) \\
&= \Re\left(\tilde{\mathbf{X}}^{m}\right) \\
&-\varepsilon^{m+1} \mathbf{W}_{m+1}^{\prime}(t)-\varepsilon^{m+2} \mathbf{Y}_{m+2}^{\prime}(t, \varepsilon)+\varepsilon^{m+1}\left[A_{\mathrm{I}}+A_{\mathrm{II}}\right] \mathbf{W}_{m+1}(t) \\
&+\varepsilon^{m+2}\left[A_{\mathrm{I}}+A_{\mathrm{II}}\right] \mathbf{Y}_{m+2}(t, \varepsilon)+\left.\varepsilon^{m+2}\left[\left[\mathbf{G}(\mathbf{X}, t, \varepsilon)_{\mathbf{X}}\right] \mathbf{W}_{m+1}\right]_{\mathbf{X}}\left[\mathbf{W}_{1}+\mathbf{Y}_{1}\right]\right|_{\mathbf{X}=\mathbf{v}} \\
&+O\left(\varepsilon^{m+3}\right) .
\end{aligned}
$$

By our specifications for $\mathbf{Y}_{m+2}$ and $\mathbf{W}_{m+1}$, the second line of the last expression for $\mathfrak{N}\left(\tilde{\mathbf{X}}^{m+1}\right)$ combines with the strictly $O\left(\varepsilon^{m+1}\right)$ terms of $\Re\left(\tilde{\mathbf{X}}^{m}\right)$ to give a term of the form

$$
\varepsilon^{m+2} \hat{\mathbf{f}}_{\mathrm{I}}(t, \varepsilon)
$$

where $\hat{\mathbf{f}}_{\mathrm{I}}$ is strictly oscillatory of class $(p-m-2)$.

The three additional assumptions of the theorem guarantee that the other terms are likewise decomposable. The smoothness conditions for these terms are satisfied since the terms must be algebraic combinations of terms which meet those requirements. Thus, provided the conditions of the theorem are met, we have decomposability to the next order.

5. Solution by Formal Expansion/Computational Examples. Under the assumptions of the preceding section, one can approximate the solution of the system (2.15) by an expansion whose terms are solutions of equations which can be treated by standard numerical techniques. In principle Theorem [4.3] can be applied repeatedly until the decomposability argument breaks down. However, provided the asymptotic form of the solution is guaranteed, one can generate the corresponding equations by a formal procedure so that repeated linearizations are unnecessary. First we introduce a fast time scale

$$
\zeta=t / \varepsilon, \quad \mathbf{X}^{\prime}=\mathbf{X}_{t}+(1 / \varepsilon) \mathbf{X}_{\zeta},
$$

and we accordingly reformulate the system (2.15):

$$
\begin{gathered}
\mathbf{X}^{\prime}=\mathbf{g}_{\mathrm{I}}(\mathbf{X}, t, \zeta, \varepsilon)+\mathbf{g}_{\mathrm{II}}(\mathbf{X}, t)+\mathbf{f}_{\mathrm{I}}(t, \zeta, \varepsilon)+\mathbf{f}_{\mathrm{II}}(t), \\
\mathbf{X}(0, \varepsilon)=\hat{\mathbf{X}}_{0}, \quad 0<\varepsilon \ll 1,0<t<T,
\end{gathered}
$$

with the appropriate modifications of the conditions on the coefficients:

(i) $\hat{\mathbf{X}}_{0}$ is independent of $\varepsilon$;

(ii) $g_{\mathrm{I}}(\mathbf{X}, t, \zeta, \varepsilon)^{(i)}=\Sigma_{j} a_{i j}(t) \exp \left[B_{i j}(\varepsilon \zeta) / \varepsilon\right] p_{i j}(\mathbf{X})$.

(iii) $g_{\mathrm{II}}(\mathbf{X}, t)^{(i)}=\sum_{j} d_{i j}(t) q_{i j}(\mathbf{X})$.

(iv) $f_{\mathrm{I}}(t, \zeta, \varepsilon)^{(i)}=\sum_{j} c_{i j}(t) \exp \left[G_{i j}(\varepsilon \zeta) / \varepsilon\right]$.

(v) $f_{\mathrm{II}}(t)^{(i)}=h_{i}(t)$.

(vi) $\left\{a_{i j}(t), d_{i j}(t), c_{i j}(t), h_{i}(t)\right\} \subset C^{p}(t)$. 
$D_{i j}(\mathbf{X})$ and $q_{i j}(\mathbf{X})$ are monomials of positive degree in the components of $\mathbf{X}$; $B_{i j}(\varepsilon \zeta) / \varepsilon$ and $G_{i j}(\varepsilon \zeta) / \varepsilon$ can be represented by $n$-vector dot products of the form

$$
\mathbf{N}^{T} \mathbf{P}(\varepsilon \zeta) / \varepsilon,
$$

where $\mathbf{N}$ is a constant $n$-vector with integral components and

$$
P(\varepsilon \zeta)^{(i)}=\int_{0}^{\xi \zeta} \lambda_{i}(s) d s, \quad \mathbf{P}^{\prime}(\varepsilon \zeta)=\boldsymbol{\Lambda}(\varepsilon \zeta), \quad\left|\mathbf{N}^{T} \boldsymbol{\Lambda}(\varepsilon \zeta)\right|>K
$$

for some positive $K$.

To extend the nomenclature of the previous section we call terms of the form (iv) strictly oscillatory (of class $p$ ), and we call terms of the form (iii) strictly nonoscillatory ( of class $p$ ). As before, subscript I denotes a strictly oscillatory function and subscript II denotes a strictly nonoscillatory function. The leading-order antiderivative of an oscillatory function is given by the linear operator

$$
\tilde{L}\{\mathbf{c}(t) \exp [B(\varepsilon \zeta) / \varepsilon]\}=\left(\mathbf{c}(t) / B^{\prime}(t)\right) \exp [B(\varepsilon \zeta) / \varepsilon] \text {. }
$$

To maintain our formalism we must insist that $\zeta$-dependence occur only as in (iv). Thus, we must interpret the $\zeta$-derivative of an oscillatory function by the rule

$$
\frac{\partial}{\partial \zeta}(\mathbf{c}(t) \exp [B(\varepsilon \zeta) / \varepsilon])=\mathbf{c}(t) B^{\prime}(t) \exp [B(\varepsilon \zeta) / \varepsilon]
$$

whereby we have

$$
\begin{aligned}
\frac{\partial}{\partial \zeta} \tilde{E}\{\mathbf{c}(t) \exp [B(\varepsilon \zeta) / \varepsilon]\} & =\mathbf{c}(t) \exp [B(\varepsilon \zeta) / \varepsilon], \\
\tilde{\mathcal{E}}\left\{\frac{\partial}{\partial \zeta} \mathbf{c}(t) \exp [B(\varepsilon \zeta) / \varepsilon]\right\} & =\mathbf{c}(t) \exp [B(\varepsilon \zeta) / \varepsilon] .
\end{aligned}
$$

This procedure apparently does not correspond to a traditional multiscaling argument; however, we are only attempting to derive a set of formal rules which mimic the balancing arguments of Section 4. The following assumptions give justification to our methodology.

Assumption [5A]. The reduced system

$$
\mathbf{V}^{\prime}=\mathbf{g}_{\mathrm{II}}(\mathbf{V}, t)+\mathbf{f}_{\mathrm{II}}(t), \quad \mathbf{V}(0)=\hat{\mathbf{X}}_{0},
$$

is well-posed and has a bounded solution in $C^{p+1}(t)$.

Assumption [5B]. Theorem [4.3] can be successively applied to system (2.15) to give an asymptotic expansion for $\mathbf{X}(t, \varepsilon)$ :

$$
\begin{gathered}
\mathbf{X}=\sum_{k=0}^{m} \mathbf{X}_{k} \varepsilon^{k}+\varepsilon^{m+1} \mathbf{Y}_{m+1}(t, \zeta, \varepsilon)+O\left(\varepsilon^{m+1}\right) \quad(m<p), \\
\mathbf{X}_{k}=\mathbf{Y}_{k}(t, \zeta, \varepsilon)+\mathbf{W}_{k}(t) .
\end{gathered}
$$

These assumptions assure that our formalism will not break down since our procedure is really a reworking of the decomposability argument of Theorem [4.3]. In correspondence to (4.2) let $A_{\mathrm{I}}(t, \zeta, \varepsilon)$ and $A_{\mathrm{II}}(t)$ be $(n \times n)$ matrices whose $(m 1, m 2)$ components are given by

$$
\begin{gathered}
{\left[A_{\mathrm{I}}(t, \zeta, \varepsilon)\right]^{\left(m_{1} m_{2}\right)}=\sum_{j} a_{m_{1} j}(t) \exp \left[B_{m_{1} j}(\varepsilon \zeta) / \varepsilon\right]\left[P_{m_{\mathrm{L}} j}\left(\mathbf{X}_{0}\right)_{\mathbf{X}}\right]^{\left(m_{2}\right)},} \\
{\left[A_{\mathrm{II}}(t)\right]^{\left(m_{1} m_{2}\right)}=\sum_{j} d_{m_{1} j}(t)\left[q_{m_{1} j}\left(\mathbf{X}_{0}\right)_{\mathbf{X}}\right]^{\left(m_{2}\right)}}
\end{gathered}
$$


The substitution of the expansion into the differential equation gives

$$
\begin{aligned}
\left(\mathbf{X}_{0_{t}}+\mathbf{X}_{1_{\zeta}}\right)+\varepsilon\left(\mathbf{X}_{1_{t}}+\mathbf{X}_{2_{\xi}}\right)+\varepsilon^{2}\left(\mathbf{X}_{2,}+\mathbf{X}_{3_{\xi}}\right)+\cdots \\
=\mathbf{g}_{\mathrm{I}}\left(\mathbf{X}_{0}+\varepsilon \mathbf{X}_{1}+\varepsilon^{2} \mathbf{X}_{2}+\cdots, t, \zeta, \varepsilon\right)+\mathbf{f}_{\mathrm{I}}(t, \zeta, \varepsilon) \\
+\mathbf{g}_{\mathrm{II}}\left(\mathbf{X}_{0}+\varepsilon \mathbf{X}_{1}+\varepsilon \mathbf{X}_{2}+\cdots, t\right)+\mathbf{f}_{\mathrm{II}}(t)
\end{aligned}
$$

We now adopt a formal procedure to solve the system (5.11). First we expand each monomial as a power series in $\varepsilon$; then we balance successive powers of $\varepsilon$. Given that on the $k$ th level we have previously determined $\mathbf{X}_{0}, \mathbf{X}_{1}, \ldots, \mathbf{X}_{k-1}$ and $\mathbf{Y}_{k}$, we balance the $O\left(\varepsilon^{k}\right)$ terms by the following rules:

I. Determine $\mathbf{W}_{k}(t)$ to eliminate all terms which are strictly nonoscillatory. This is essentially a secularity condition designed to eliminate powers of $\zeta$ in the expansion. The appropriate initial conditions are:

$$
\mathbf{W}_{0}(0)=\hat{\mathbf{X}}_{0}, \quad \mathbf{W}_{k}(0)=-\mathbf{Y}_{k}(0,0, \varepsilon) \quad(k>0) .
$$

II. Determine $\mathbf{Y}_{k+1}(t, \zeta, \varepsilon)$ by (5.7) to balance the remaining terms.

We now illustrate this approach with two computational examples from the theory of nonlinear oscillations. For illustrative purposes we include the resulting algebra although, as we have previously noted, the analytical manipulations are conceptually simple enough to be computationally feasible. First we return to the nonlinear oscillator of Example (1) and calculate the leading order approximation:

$$
\begin{gathered}
\left(\begin{array}{l}
x_{1} \\
x_{2}
\end{array}\right)=\tilde{\mathbf{V}}+\varepsilon\left(\tilde{\mathbf{W}}_{1}+\tilde{\mathbf{Y}}_{1}\right)+\varepsilon^{2} \tilde{\mathbf{Y}}_{2}+O\left(\varepsilon^{2}\right)=\tilde{\mathbf{V}}+\varepsilon\left(\tilde{\mathbf{W}}_{1}+\tilde{\mathbf{Y}}_{1}\right)+O\left(\varepsilon^{2}\right) \\
\tilde{\mathbf{V}}=\left(\begin{array}{c}
V \\
\bar{V}
\end{array}\right), \quad \tilde{\mathbf{W}}_{1}=\left(\begin{array}{c}
W_{1} \\
\bar{W}_{1}
\end{array}\right), \quad \tilde{\mathbf{Y}}_{1}=\left(\begin{array}{c}
Y_{1} \\
\bar{Y}_{1}
\end{array}\right)
\end{gathered}
$$

In the notation of the section we have

$$
\begin{gathered}
g_{\mathrm{I}}(\mathbf{X}, t, \zeta, \varepsilon)^{(1)}=-\frac{1}{2} \exp (2 i \zeta) x_{2}-\frac{1}{24} \exp (4 i \zeta) x_{2}^{3} \\
+\frac{1}{x} \exp (2 i \zeta) x_{2}^{2} x_{1}+\frac{1}{24} \exp (-2 i \zeta) x_{1}^{3} \\
g_{\mathrm{I}}(\mathbf{X}, t, \zeta, \varepsilon)^{(2)}=\bar{g}_{\mathrm{I}}(\mathbf{X}, t, \zeta, \varepsilon)^{(1)} \\
g_{\mathrm{II}}(\mathbf{X}, t)^{(1)}=\frac{1}{2} x_{1}-\frac{1}{8} x_{2} x_{1}^{2}, \quad g_{\mathrm{II}}(\mathbf{X}, t)^{(2)}=\bar{g}_{\mathrm{II}}(\mathbf{X}, t)^{(1)}
\end{gathered}
$$

The appropriate version of $(5.11)$ is

$$
\begin{aligned}
& \tilde{\mathbf{V}}_{t}+\tilde{\mathbf{Y}}_{1_{\zeta}}+\varepsilon\left(\tilde{\mathbf{Y}}_{1_{t}}+\tilde{\mathbf{W}}_{1_{t}}+\mathbf{Y}_{2_{\zeta}}\right)+O\left(\varepsilon^{2}\right) \\
& =\mathbf{g}_{\mathrm{I}}(\mathbf{V}, t, \zeta, \varepsilon)+\mathbf{g}_{\mathrm{II}}(\mathbf{V}, t)+\varepsilon\left(A_{\mathrm{I}} \tilde{\mathbf{W}}_{1}+A_{\mathrm{II}} \tilde{\mathbf{W}}_{1}+A_{\mathrm{I}} \tilde{\mathbf{Y}}_{1}+A_{\mathrm{II}} \tilde{\mathbf{Y}}_{1}\right)+O\left(\varepsilon^{2}\right) .
\end{aligned}
$$

The first row of $A_{\mathrm{I}}$ is the $(1 \times 2)$ matrix $\mathcal{U}_{1}(t, \zeta, \varepsilon)$ where

$$
Q_{I}(t, \zeta, \varepsilon)=\left[\begin{array}{c}
{\left[\frac{1}{8} \exp (2 i \zeta) \bar{V}^{2}+\frac{1}{8} \exp (-2 i \zeta) V^{2}\right]} \\
{\left[-\frac{1}{2} \exp (2 i \zeta)-\frac{1}{8} \exp (4 i \zeta) \bar{V}^{2}+\frac{1}{4} \exp (2 i \zeta) \bar{V} V\right]}
\end{array}\right]^{T}
$$

and likewise the first row of $A_{\mathrm{II}}(t)$ is the $(1 \times 2)$ matrix $\mathbb{Q}_{\mathrm{II}}(t)$ where

$$
Q_{\mathrm{II}}(t)=\left[\begin{array}{c}
\frac{1}{2}-\frac{1}{4} \bar{V} V \\
-\frac{1}{8} V^{2}
\end{array}\right]^{T}
$$


By Rule I we determine $V(t)$ as the solution of

$$
V^{\prime}=\frac{1}{2} V-\frac{1}{8} \bar{V} V^{2}, \quad V(0)=1,
$$

and by Rule II $Y_{1}$ is given by

$$
\begin{aligned}
Y_{1}(t, \zeta, \varepsilon)= & \tilde{\mathcal{L}}\left\{-\frac{1}{2} \exp (2 i \zeta) \bar{V}-\frac{1}{24} \exp (4 i \zeta) \bar{V}^{3}\right. \\
& \left.+\frac{1}{8} \exp (2 i \zeta) \bar{V}^{2} V+\frac{1}{24} \exp (-2 i \zeta) V^{3}\right\} \\
= & \frac{i}{4} \exp (2 i \zeta) \bar{V}+\frac{i}{96} \exp (4 i \zeta) \bar{V}^{3}+\frac{-1}{16} \exp (2 i \zeta) \bar{V}^{2} V+\frac{i}{48} \exp (-2 i \zeta) V^{3}
\end{aligned}
$$

With the exception of $A_{\mathrm{I}} \tilde{\mathbf{Y}}_{1}$ all $O(\varepsilon)$ terms of (5.15) are either strictly oscillatory or strictly nonoscillatory. We have

$$
\begin{gathered}
\Theta_{\mathrm{I}}\left(\begin{array}{c}
Y_{1} \\
\bar{Y}_{1}
\end{array}\right)=R(V)+\langle\text { strictly oscillatory terms }\rangle, \\
R(V)=\frac{i}{8} V+\frac{3 i}{256} \bar{V}^{2} V^{3}-\frac{i}{16} \bar{V}^{2} V,
\end{gathered}
$$

and thus by Rule I the system for $W_{1}$ is

$$
W_{1}^{\prime}=\Theta_{\mathrm{II}}\left(\begin{array}{l}
W_{1} \\
\bar{W}_{1}
\end{array}\right)+R(V), \quad W_{1}(0)=-Y_{1}(0,0, \varepsilon)=-7 i / 32
$$

By using (5.1), we can restore the full $t$-dependence of the coefficients; in terms of the original variables we then have

$$
z_{1}=\left.\operatorname{Re}\left\{\exp (-i \zeta) x_{1}\right\}\right|_{\zeta=t / \varepsilon}, \quad z_{2}=\left.\operatorname{Im}\left\{\exp (-i \zeta) x_{2}\right\}\right|_{\zeta=t / \varepsilon} .
$$

Using this analysis, we outline an approximation scheme based on standard discretization techniques. First we ignore $O\left(\varepsilon^{2}\right)$ terms of the expansion. Using a step size $h$, we approximate the solution of Eq. (5.18) by means of a fourth-order Runge-Kutta scheme (see Lambert [15, p. 126]); then $Y_{1}$ is given explicitly by (5.19), and with the same step size $h$ we approximate $W_{1}$ from (5.21) by means of a forward Euler predictor followed by two trapezoidal rule correctors (see Lambert [15, p. 85]). The total error for this approximation is then

$$
O\left(h^{4}\right)+O\left(\varepsilon^{2}\right)+O\left(\varepsilon h^{2}\right) .
$$

Computations were done with single-precision accuracy on a VAX11/780 for the case

$$
\varepsilon=.01, \quad h=.1,
$$

and, therefore, by (5.23) one might expect a grid error of approximately $10^{-3}$.

In phase space the solutions of (2.6) approach a stable limit cycle of approximate radius two. Thus, in Figure 1 we plot the resulting approximation to the amplitude

$$
A(t)=\left[z_{1}^{2}+z_{2}^{2}\right]^{1 / 2}
$$

as a function of the rescaled time variable $t$, and also we plot the approximate curve of

$$
z_{1}(t)=y(t / \varepsilon)
$$

in Figure 2. In both cases we have linearly interpolated the amplitudes and the phases between grid points to achieve full plotting accuracy. In the second two 
columns of Table 2 we compare the computed grid values with the accepted function values, which were computed with double-precision accuracy by means of a fourthorder Runge-Kutta scheme with a time step

$$
h=10^{-4} \text {. }
$$

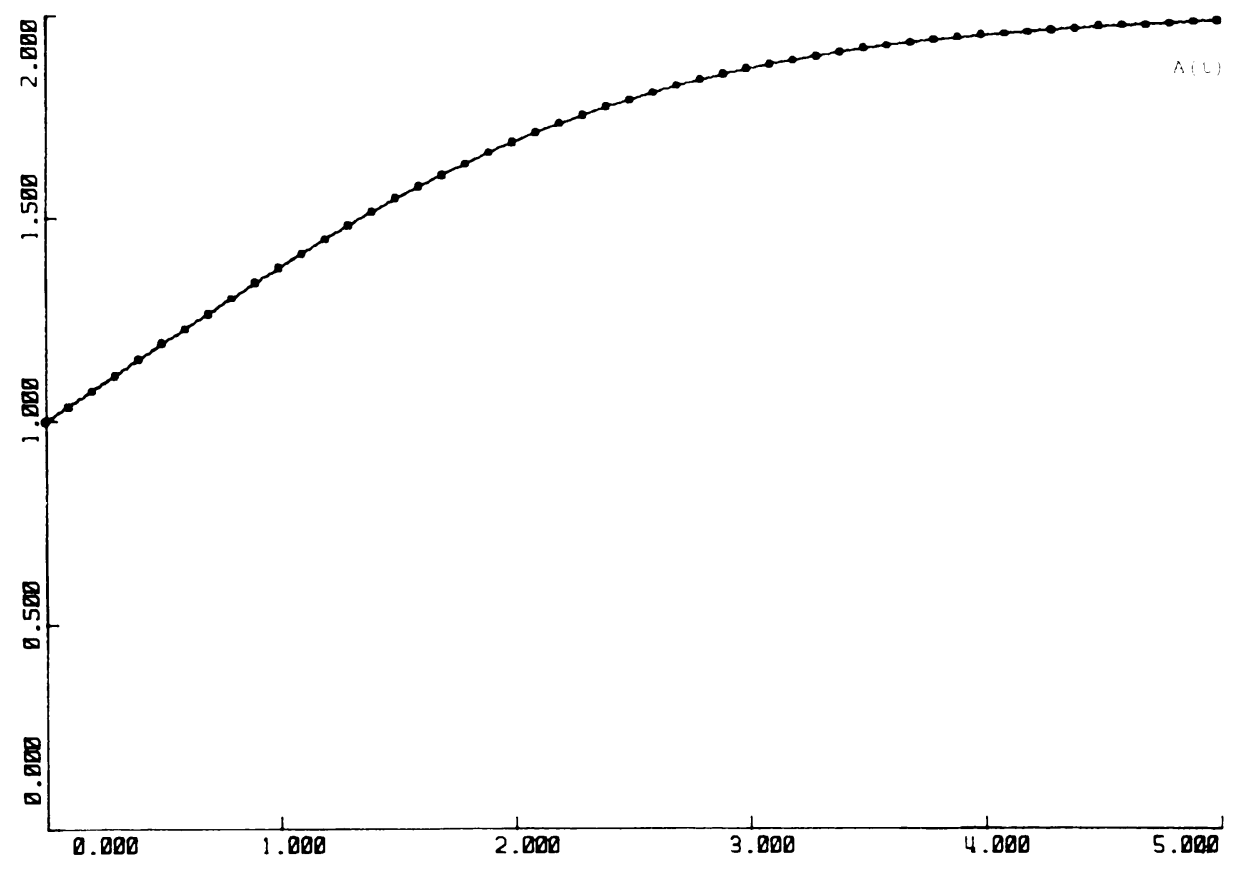

FIGURE 1

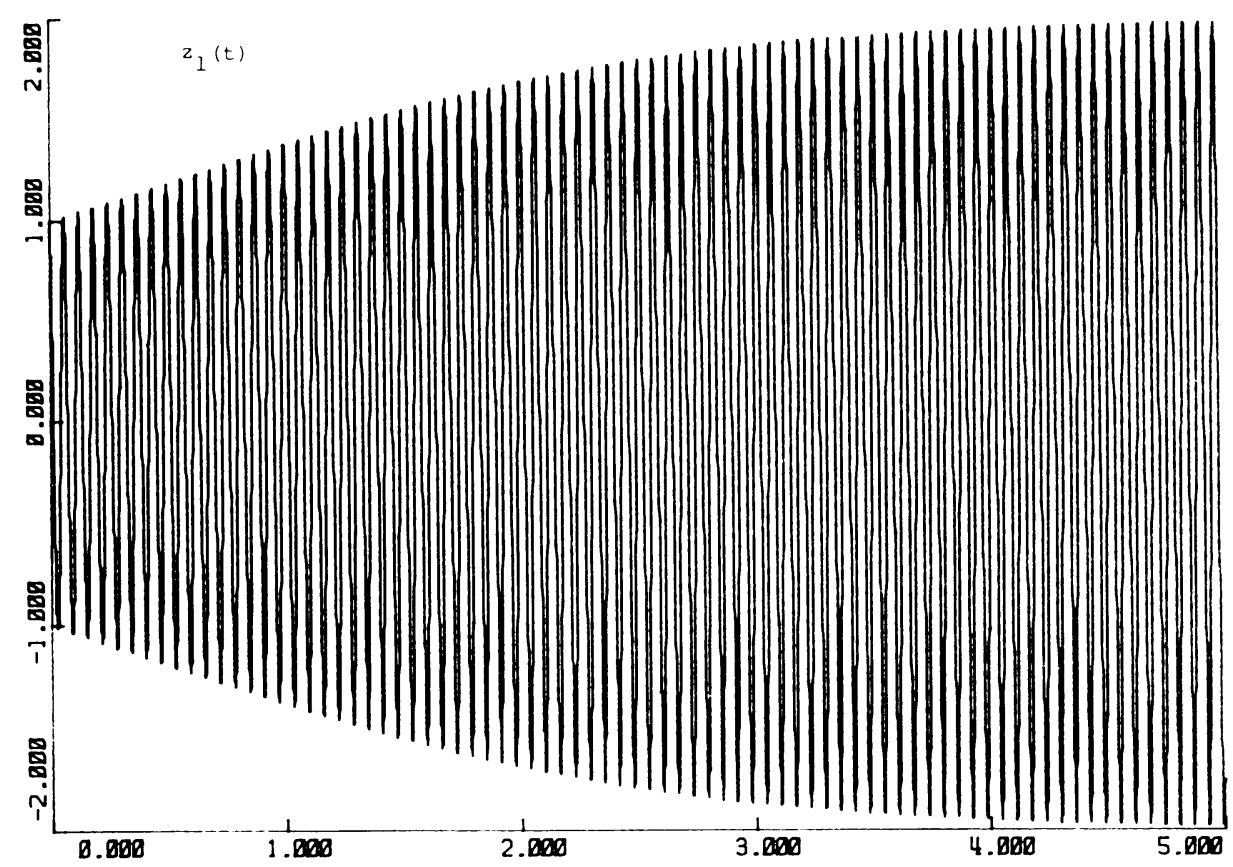

FigURE 2 
TABLE 2

\begin{tabular}{|c|c|c|c|c|c|}
\hline$N$ & $t_{1}$ & $\operatorname{ERR}(A)$ & $\begin{array}{l}\operatorname{ERR}\left(z_{1}\right) \\
\quad(\text { absolu }\end{array}$ & $\begin{array}{l}\operatorname{ERR}\left(\tilde{E}_{1}\right) \\
\text { errors) }\end{array}$ & $\operatorname{ERR}\left(\tilde{E}_{2}\right)$ \\
\hline 0 & 0.00 & $0.0 \mathrm{E}+00$ & $0.0 \mathrm{E}+00$ & $0.0 \mathrm{E}+00$ & $0.0 \mathrm{E}+00$ \\
\hline 2 & 0.20 & $0.7 \mathrm{E}-06$ & $0.1 \mathrm{E}-05$ & $0.7 \mathrm{E}-04$ & $0.2 \mathrm{E}-04$ \\
\hline 4 & 0.40 & $0.1 E-05$ & $0.4 \mathrm{E}-05$ & $0.2 \mathrm{E}-03$ & $0.6 \mathrm{E}-04$ \\
\hline 6 & 0.60 & $0.3 \mathrm{E}-05$ & $0.4 \mathrm{E}-05$ & $0.1 E-03$ & $0.6 E-04$ \\
\hline 8 & 0.80 & $0.4 \mathrm{E}-05$ & $0.1 \mathrm{E}-04$ & $0.4 \mathrm{E}-04$ & $0.3 E-04$ \\
\hline 10 & 1.00 & $0.4 \mathrm{E}-05$ & $0.2 E-05$ & $0.5 \mathrm{E}-04$ & $0.4 \mathrm{E}-05$ \\
\hline 12 & 1.20 & $0.4 \mathrm{E}-05$ & $0.1 E-04$ & $0.2 \mathrm{E}-03$ & $0.1 E-03$ \\
\hline 14 & 1.40 & $0.7 \mathrm{E}-05$ & $0.3 \mathrm{E}-05$ & $0.7 \mathrm{E}-04$ & $0.2 \mathrm{E}-03$ \\
\hline 16 & 1.60 & $0.8 \mathrm{E}-05$ & $0.2 \mathrm{E}-05$ & $0.2 E-04$ & $0.4 \mathrm{E}-03$ \\
\hline 18 & 1.80 & $0.3 \mathrm{E}-05$ & $0.3 E-04$ & $0.7 \mathrm{E}-04$ & $0.2 \mathrm{E}-02$ \\
\hline 20 & 2.00 & $0.4 \mathrm{E}-06$ & $0.3 \mathrm{E}-05$ & $0.3 \mathrm{E}-03$ & $0.3 \mathrm{E}-02$ \\
\hline 22 & 2.20 & $0.1 E-04$ & $0.1 E-04$ & $0.7 \mathrm{E}-03$ & $0.2 \mathrm{E}-02$ \\
\hline 24 & 2.40 & $0.6 \mathrm{E}-05$ & $0.3 E-04$ & $0.2 \mathrm{E}-02$ & $0.1 E-02$ \\
\hline 26 & 2.60 & $0.1 E-04$ & $0.2 \mathrm{E}-05$ & $0.3 E-02$ & $0.2 \mathrm{E}-02$ \\
\hline 28 & 2.80 & $0.1 \mathrm{E}-05$ & $0.1 \mathrm{E}-04$ & $0.4 \mathrm{E}-02$ & $0.1 \mathrm{E}-02$ \\
\hline 30 & 3.00 & $0.1 E-04$ & $0.8 \mathrm{E}-05$ & $0.5 \mathrm{E}-02$ & $0.1 E-02$ \\
\hline 32 & 3.20 & $0.1 \mathrm{E}-04$ & $0.2 \mathrm{E}-04$ & $0.6 \mathrm{E}-02$ & $0.1 \mathrm{E}-02$ \\
\hline 34 & 3.40 & $0.1 \mathrm{E}-04$ & $0.5 \mathrm{E}-04$ & $0.6 \mathrm{E}-02$ & $0.1 E-03$ \\
\hline 36 & 3.60 & $0.6 \mathrm{E}-05$ & $0.7 E-04$ & $0.6 \mathrm{E}-02$ & $0.2 E-03$ \\
\hline 38 & 3.80 & $0.2 E-04$ & $0.1 \mathrm{E}-04$ & $0.7 \mathrm{E}-02$ & $0.2 \mathrm{E}-03$ \\
\hline 40 & 4.00 & $0.6 \mathrm{E}-05$ & $0.1 E-04$ & $0.6 \mathrm{E}-02$ & $0.2 \mathrm{E}-03$ \\
\hline 42 & 4.20 & $0.8 \mathrm{E}-05$ & $0.5 E-04$ & $0.5 \mathrm{E}-02$ & $0.2 E-03$ \\
\hline 44 & 4.40 & $0.2 \mathrm{E}-04$ & $0.2 \mathrm{E}-04$ & $0.5 \mathrm{E}-02$ & $0.9 \mathrm{E}-03$ \\
\hline 46 & 4.60 & $0.6 \mathrm{E}-05$ & $0.5 E-04$ & $0.3 \mathrm{E}-02$ & $0.8 \mathrm{E}-03$ \\
\hline 48 & 4.80 & $0.1 E-04$ & $0.3 E-04$ & $0.2 \mathrm{E}-02$ & $0.1 E-02$ \\
\hline 50 & 5.00 & $0.1 E-04$ & $0.5 E-04$ & $0.8 \mathrm{E}-03$ & $0.3 \mathrm{E}-02$ \\
\hline
\end{tabular}

One likewise can apply these techniques to systems of coupled nonlinear oscillators. The following extensively analyzed system is taken from the theory of stellar orbits in a galaxy (see, for example, Kevorkian and Cole [12]):

$$
\begin{gathered}
r_{1}^{\prime \prime}+a^{2} r_{1}=\varepsilon r_{2}^{2}, \quad r_{2}^{\prime \prime}+b^{2} r_{2}=2 \varepsilon r_{1} r_{2}, \quad r_{1}(0, \varepsilon)=1, \quad r_{2}(0, \varepsilon)=1, \\
r_{1}^{\prime}(0, \varepsilon)=0, \quad r_{2}^{\prime}(0, \varepsilon)=0, \quad 0<\varepsilon \ll 1,0<\tilde{t}<T / \varepsilon .
\end{gathered}
$$

Here $r_{1}$ stands for the radial displacement of the orbit of a star from a reference circular orbit, and $r_{2}$ stands for the deviation of the orbit from the galactic plane. With the change of variables

$$
\mathbf{Z}=\left[z_{1}, z_{2}, z_{3}, z_{4}\right]^{T}=\left[r_{1}, r_{1}^{\prime} / a, r_{2}, r_{2}^{\prime} / b\right]^{T}, \quad t=\varepsilon \tilde{t},
$$


we have

$$
\begin{aligned}
& \mathbf{Z}^{\prime}=(1 / \varepsilon)\left[\begin{array}{cccc}
0 & a & 0 & 0 \\
-a & 0 & 0 & 0 \\
0 & 0 & 0 & b \\
0 & 0 & -b & 0
\end{array}\right] \mathbf{Z}+\left(\begin{array}{c}
0 \\
(1 / a) z_{3}^{2} \\
0 \\
(2 / b) z_{1} z_{3}
\end{array}\right) \\
& \mathbf{Z}(0, \varepsilon)=[1,0,1,0]^{T}, \quad 0<t<T, \quad 0<\varepsilon \ll 1 .
\end{aligned}
$$

By a transformation similar to (2.7), we can reduce the system to diagonal form. Thus after the change of variables

$$
\mathbf{Z}=\tilde{S} \mathbf{Z}, \quad \mathbf{U}=\left[u_{1}, u_{2}, u_{3}, u_{4}\right]^{T} . \quad \tilde{S}=\left[\begin{array}{cc}
\hat{S} & 0 \\
0 & \hat{S}
\end{array}\right], \quad \hat{S}=(1 / 2)\left[\begin{array}{cc}
1 & 1 \\
-i & i
\end{array}\right] .
$$

the equations become

$$
\begin{gathered}
\mathbf{U}^{\prime}=(i / \varepsilon)\left[\begin{array}{cccc}
-a & 0 & 0 & 0 \\
0 & a & 0 & 0 \\
0 & 0 & -b & 0 \\
0 & 0 & 0 & b
\end{array}\right] \mathbf{U}+\left[\begin{array}{c}
f_{1}(\mathbf{U}) \\
-f_{1}(\mathbf{U}) \\
f_{2}(\mathbf{U}) \\
-f_{2}(\mathbf{U})
\end{array}\right), \\
\mathbf{U}(0, \varepsilon)=[1,1,1,1]^{T}, \quad 0<t<T, 0<\varepsilon \ll 1, \\
f_{1}(\mathbf{U})=i\left(u_{3}+u_{4}\right)^{2} / 4 a=(i / 4 a)\left[u_{3}^{2}+2 u_{3} u_{4}+u_{4}^{2}\right], \\
f_{2}(\mathbf{U})=i\left(u_{1}+u_{2}\right)\left(u_{3}+u_{4}\right) / 2 b=(i / 2 b)\left[u_{1} u_{3}+u_{1} u_{4}+u_{2} u_{3}+u_{2} u_{4}\right] .
\end{gathered}
$$

As in (2.10), we now factor out the leading-order oscillatory behavior by means of the transformation

$$
\begin{gathered}
\mathbf{U}=S(t, \varepsilon) \mathbf{X}, \quad \mathbf{X}=\left[x_{1}, x_{2}, x_{3}, x_{4}\right]^{T}, \\
S(t, \varepsilon)=\operatorname{diag}[\exp (-i a t / \varepsilon), \exp (i a t / \varepsilon), \exp (-i b t / \varepsilon), \exp (i b t / \varepsilon)] .
\end{gathered}
$$

and obtain the system

$$
\begin{gathered}
\mathbf{X}^{\prime}=\left[\begin{array}{c}
\exp (i a t / \varepsilon) f_{1}(S(t, \varepsilon) \mathbf{X}) \\
-\exp (-i a t / \varepsilon) f_{1}(S(t, \varepsilon) \mathbf{X}) \\
\exp (i b t / \varepsilon) f_{2}(S(t, \varepsilon) \mathbf{X}) \\
-\exp (-i b t / \varepsilon) f_{2}(S(t, \varepsilon) \mathbf{X})
\end{array}\right] \\
\mathbf{X}(0, \varepsilon)=[1,1,1,1]^{T}, \quad 0<t<T, 0<\varepsilon \ll 1 .
\end{gathered}
$$

From the structure of the transformations we have

$$
x_{2}=\bar{x}_{1}, \quad x_{4}=\bar{x}_{3},
$$

and therefore, similarly to the first example, we have replaced the original four-dimensional real system with a two-dimensional complex system. Once again, in the spirit of Section 4, we introduce an asymptotic expansion in powers of $\varepsilon$ :

$$
\mathbf{X}=\tilde{\mathbf{V}}+\varepsilon\left(\tilde{\mathbf{W}}_{1}+\tilde{\mathbf{Y}}_{1}\right)+O\left(\varepsilon^{2}\right)
$$

$$
\tilde{\mathbf{V}}=\left[V_{A}, \bar{V}_{A}, V_{B}, \bar{V}_{B}\right]^{T}, \quad \tilde{\mathbf{W}}_{1}=\left[W_{A}, \bar{W}_{A}, W_{B}, \bar{W}_{B}\right]^{T}, \quad \tilde{\mathbf{Y}}_{1}=\left[Y_{A}, \bar{Y}_{A}, Y_{B}, \bar{Y}_{B}\right]^{T} .
$$


Here the fast scale is again given by (5.1). The most interesting resonances occur for the case

$$
a=2 b,
$$

and so we consider the parameter values

$$
a=1, \quad b=.5 .
$$

System (5.34) then has the form (5.2) with

$$
\begin{aligned}
& g_{\mathrm{I}}^{(1)}=(i / 2) x_{3} x_{4} \exp (i \zeta)+(i / 4) x_{4}^{2} \exp (2 i \zeta), \quad g_{\mathrm{I}}^{(2)}=\overline{g_{\mathrm{I}}^{(1)}}, \\
& g_{\mathrm{I}}^{(3)}=i x_{1} x_{3} \exp (-i \zeta)+i x_{2} x_{3} \exp (i \zeta)+i x_{2} x_{4} \exp (2 i \zeta), \\
& g_{\mathrm{I}}^{(4)}=\overline{g_{\mathrm{I}}^{(3)}}, \quad g_{\mathrm{II}}^{(1)}=(i / 4) x_{3}^{2}, \\
& g_{\mathrm{II}}^{(2)}=\overline{g_{\mathrm{II}}^{(1)}}, \quad g_{\mathrm{II}}^{(3)}=i x_{1} x_{4}, \quad g_{\mathrm{II}}^{(4)}=\overline{g_{\mathrm{II}}^{(3)}} .
\end{aligned}
$$

We proceed as in the first example by applying the balancing arguments of Section 4 . The first and third rows of $A_{\text {II }}$ are given by the $(2 \times 4)$ matrix $\mathbb{Q}_{\text {II }}$, where

$$
\mathbb{U}_{\mathrm{II}}=\left[\begin{array}{cc}
0 & i \bar{V}_{B} \\
0 & 0 \\
(i / 2) V_{B} & 0 \\
0 & i V_{A}
\end{array}\right]^{T}
$$

The first and third rows of $A_{\mathrm{I}}$ are given by the $(2 \times 4)$ matrix $\mathbb{Q}_{\mathrm{I}}$, where

$$
\mathbb{Q}_{\mathrm{I}}=\left[\begin{array}{cc}
0 & i V_{B} \exp (-i \zeta) \\
0 & i V_{B} \exp (i \zeta)+i \bar{V}_{B} \exp (2 i \zeta) \\
(i / 2) \bar{V}_{B} \exp (i \zeta) & i V_{A} \exp (-i \zeta)+i \bar{V}_{A} \exp (i \zeta) \\
(i / 2) V_{B} \exp (i \zeta)+(i / 2) \bar{V}_{B} \exp (2 i \zeta) & i \bar{V}_{A} \exp (2 i \zeta)
\end{array}\right]^{T}
$$

$\tilde{\mathbf{V}}$ is then determined by the solution of

$$
\left(\begin{array}{c}
V_{A} \\
V_{B}
\end{array}\right)^{\prime}=\left(\begin{array}{c}
(i / 4) V_{B}^{2} \\
(i / 2) V_{B} V_{A}
\end{array}\right), \quad\left(\begin{array}{c}
V_{A}(0, \varepsilon) \\
V_{B}(0, \varepsilon)
\end{array}\right)=\left(\begin{array}{l}
1 \\
1
\end{array}\right), \quad 0<t<T,
$$

and, as in (5.19), $\tilde{\mathbf{Y}}_{1}$ is given explicitly by

$$
\left(\begin{array}{c}
Y_{A} \\
Y_{B}
\end{array}\right)=\left(\begin{array}{c}
(1 / 2) V_{B} \bar{V}_{B} \exp (i \zeta)+(1 / 8) \bar{V}_{B}^{2} \exp (2 i \zeta) \\
-V_{B} V_{A} \exp (-i \zeta)+V_{B} \bar{V}_{A} \exp (i \zeta)+(1 / 2) \bar{V}_{B} \bar{V}_{A} \exp (2 i \zeta)
\end{array}\right)
$$

With the exception of $A_{\mathrm{I}} \tilde{\mathbf{Y}}_{1}$ all $O(\varepsilon)$ terms of (5.15) are either strictly oscillatory or strictly nonoscillatory. We have

(5.44) $Q_{\mathrm{I}}\left(\begin{array}{c}Y_{A} \\ \bar{Y}_{A} \\ Y_{B} \\ \bar{Y}_{B}\end{array}\right)=\left(\begin{array}{c}(i / 4) V_{A} V_{B} \bar{V}_{B} \\ (i / 2) V_{A} \bar{V}_{A} V_{B}+(9 i / 8) V_{B}^{2} \bar{V}_{B}\end{array}\right)+\langle$ oscillatory terms $\rangle$, 
and thus the system which determines $\tilde{\mathbf{W}}_{1}$ is

$$
\begin{gathered}
\left(\begin{array}{c}
W_{A} \\
W_{B}
\end{array}\right){ }^{\prime}=\left(\begin{array}{c}
(i / 2) V_{B} W_{B} \\
i \bar{V}_{B} W_{A}+i V_{A} \bar{W}_{B}
\end{array}\right)+\left(\begin{array}{c}
(i / 4) V_{A} V_{B} \bar{V}_{B} \\
(i / 2) V_{A} \bar{V}_{A} V_{B}+(9 i / 8) V_{B}^{2} \bar{V}_{B}
\end{array}\right), \\
\left(\begin{array}{l}
W_{A}(0) \\
W_{B}(0)
\end{array}\right)=\left(\begin{array}{l}
-Y_{A}(0,0, \varepsilon) \\
-Y_{B}(0,0, \varepsilon)
\end{array}\right)=\left(\begin{array}{l}
-5 / 8 \\
-1 / 2
\end{array}\right), \quad 0<t<T .
\end{gathered}
$$

Once again the full $t$-dependence of the system can be restored by (5.1). In the original system variables we have:

$$
\begin{array}{ll}
z_{1}=\left.\operatorname{Re}\left\{\exp (-i \zeta) x_{1}\right\}\right|_{\zeta=t / \varepsilon}, & z_{2}=\left.\operatorname{Im}\left\{\exp (-i \zeta) x_{1}\right\}\right|_{\zeta=t / \varepsilon}, \\
z_{3}=\left.\operatorname{Re}\left\{\exp (-i \zeta / 2) x_{3}\right\}\right|_{\zeta=t / \varepsilon}, & z_{4}=\left.\operatorname{Im}\left\{\exp (-i \zeta / 2) x_{3}\right\}\right|_{\zeta=t / \varepsilon} .
\end{array}
$$

Now we can apply the same approximation techniques to this system, which is characterized by the energy integral

$$
E(t)=\left(a^{2} / 2\right)\left(z_{1}^{2}+z_{2}^{2}\right)+\left(b^{2} / 2\right)\left(z_{3}^{2}+z_{4}^{2}\right)-\varepsilon z_{1} z_{3}^{2}=E_{0} .
$$

The sharing of this energy between the two oscillators is illustrated in Figure 3, where we have plotted our numerical approximations to the leading-order energy functions

$$
\tilde{E}_{1}(t)=\left(a^{2} / 2\right)\left[z_{1}^{2}+z_{2}^{2}\right], \quad \tilde{E}_{2}(t)=\left(b^{2} / 2\right)\left[z_{3}^{2}+z_{4}^{2}\right] .
$$

Once again we have linearly interpolated the amplitudes and phases between the grid points. In the last two columns of Table 2 we compare the computed grid values with the accepted function values, which were computed with double-precision accuracy by means of a fourth-order Runge-Kutta scheme with a time step

$$
h=10^{-4} \text {. }
$$

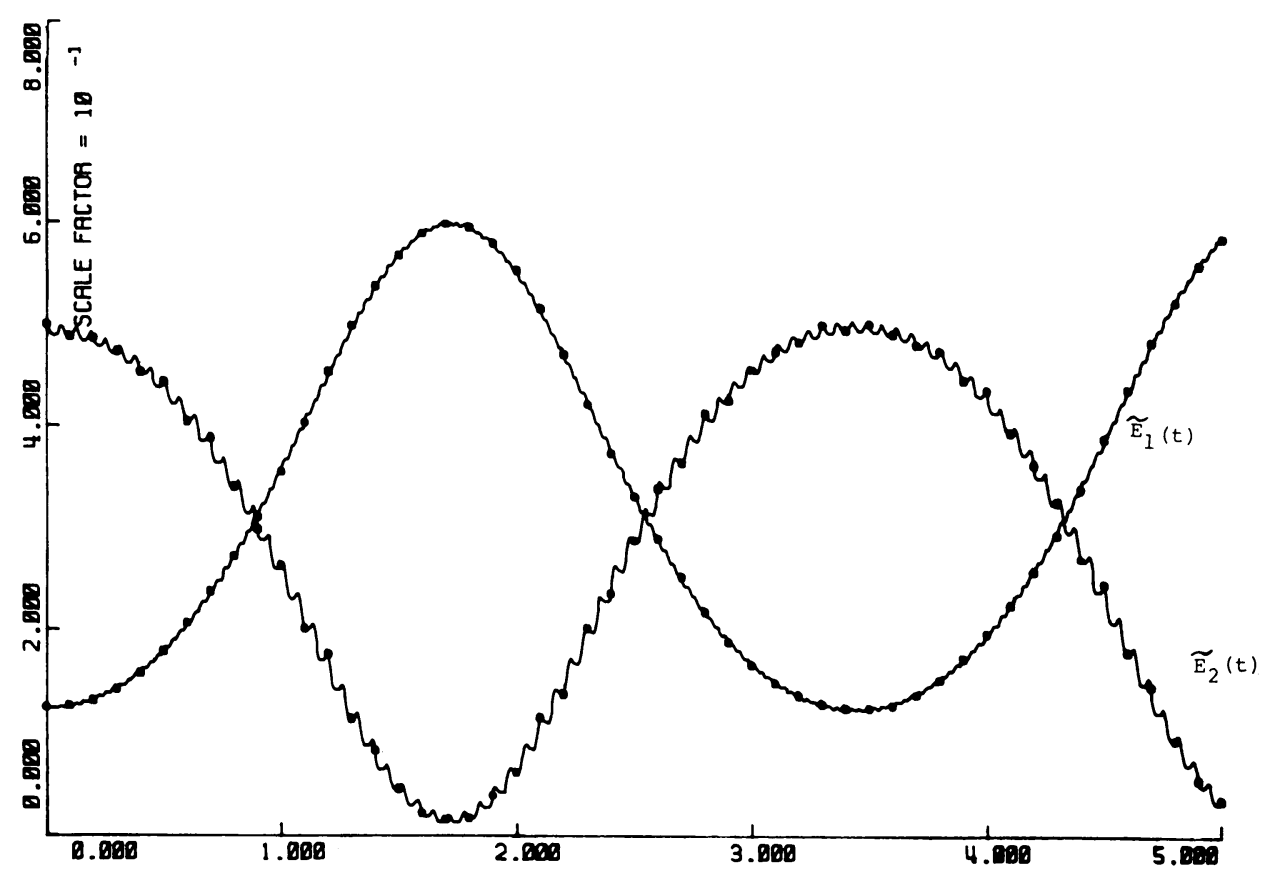

Figure 3 
The grid error, somewhat larger than in the first example, is mainly due to the truncation of the asymptotic expansion. Indeed, decreasing $\varepsilon$ by a factor of .1 caused the grid error to fall by a factor of .01 .

6. Acknowledgement. The author is indebted to Professor H.-O. Kreiss for his helpful criticism and advice.

Department of Applied Mathematics

California Institute of Technology

Pasadena, California 91125

1. V. Amdursky \& A. ZIv, On the Numerical Treatment of Stiff Highly-Oscillatory Sistems, IBM Isreal Scientific Center Technical Report No. 15, Haifa, 1974.

2. V. Amdursky \& A. ZIV, The Numerical Treatment of Linear Highly Oscillatory O. D. E. S!stems by Reduction to Non-Oscillatory Type, IBM Israel Scientific Center Report No. 39. Haifa, 1976.

3. V. Amdursky \& A. ZIV, "On the numerical solution of stiff linear systems of the oscillatory type." SIAM J. Appl. Math. , v. 33, 1977, pp. 593-606.

4. N. N. Bogoliubov \& Y. A. Mitropolsky, Asymptotic Methods in the Theory of Nonlinear Oscillations, Gordon and Breach, New York, 1961.

5. G. Browning \& H.-O. Kreiss, "Problems with different time scales for nonlinear partial differential equations,” SIAM J. Appl. Math., v. 42, 1982, pp. 704-718.

6. E. A. Coddington \& N. Levinson, Theory of Ordinary Differential Equations, McGraw-Hill, New York, 1955.

7. S. O. Fatunla, "Numerical integrators for stiff and highly oscillatory differential equations," Math. Comp., v. 34, 1980, pp. 373-390.

8. W. GautschI, "Numerical integration of ordinary differential equations based on trigonometric polynomials,” Numer. Math., v. 3, 1961, pp. 381-397.

9. C. W. GEAR, "Numerical solution of ordinary differential equations: Is there anything left to do?," SIAM Rev., v. 23, 1981, pp. 10-24.

10. O. F. GrafF \& D. G. BetTis, "Modified.multirevolution integration methods for satellite orbit computation," Celestial Mech., v. 11, 1975, pp. 433-448.

11. F. C. Hoppensteadt \& W. L. Miranker, "Differential equations having rapidly changing solutions: Analytic methods for weakly nonlinear systems," J. Differential Equations, v. 22, 1976, pp. 237-249.

12. J. Kevorkian \& J. D. Cole, Perturbation Methods in Applied Mathematics, Springer-Verlag, New York, 1981.

13. H.-O. KREISS, "Difference methods for stiff ordinary differential equations," SIAM J. Numer. Anal., v. 15, 1978, pp. 21-58.

14. H.-O. Kreiss, "Problems with different time scales for ordinary differential equations," SIAM J. Numer. Anal., v. 16, 1979, pp. 980-998.

15. J. D. Lambert, Computational Methods in Ordinary Differential Equations, Wiley, Chichester, England, 1973.

16. B. LiNDBERG, “On smoothing and extrapolation for the trapezoidal rule," BIT, v. 11, 1971, pp. $29-52$.

17. G. MAJDa, "Filtering techniques for oscillatory stiff O.D.E.'s," SIAM J. Numer. Anal. (To appear.)

18. W. L. Miranker, Numerical Methods for Stiff Equations and Singular Perturhation Prohlems, Reidel, Dordrecht, Holland, 1981.

19. W. L. Miranker \& F. Hoppensteadt, Numerical Methods for Stiff Systems of Differential Equations Related With Transistors, Tunnel Diodes, etc., Lecture Notes in Comput. Sci., Vol. 10, Springer-Verlag, New York, 1974.

20. W. L. Miranker \& M. van Veldhuizen, “The method of envelopes,” Math. Comp.,v. 32, 1978. pp. 453-498.

21. W. L. Miranker \& G. Wahba, "An averaging method for the stiff highly oscillatory problem," Math. Comp., v. 30, 1976, pp. 383-399.

22. A. Nadeau, J. GuYard \& M. R. Feix, "Algebraic-numerical method for the slightly perturbed harmonic oscillator," Math. Comp.,v. 28, 1974, pp. 1057-1066.

23. J. C. NEU, "The method of near-identity transforms and its applications," SIAM J. Appl. Math., v. 38,1980, pp. $189-200$. 
24. A. H. Nayfeh, Perturbation Methods, Wiley, New York, 1973.

25. L. R. Petzold, "An efficient numerical method for highly oscillatory ordinary differential equations," SIAM J. Numer. Anal., v. 18, 1981, pp. 455-479.

26. L. R. Petzold \& C. W. Gear, Methods for Oscillating Problems, Dept. of Computer Science File \#889. University of Illinois at Urbana-Champaign, 1977.

27. R. E. Scheid, Jr., The Accurate Numerical Solution of Highly Oscillatory Ordinary Differential Equations, Ph.D. thesis, California Institute of Technology, 1982.

28. A. D. Snyder \& G. C. Fleming, "Approximation by aliasing with applications to "Certaine" stiff differential equations," Math. Comp., v. 28, 1974, pp. 465-473.

29. C. E. Velez, Numerical Integration of Orbits in Multirevolution Steps, NASA Technical Note D-5915, Goddard Space Flight Center, Greenbelt, Maryland, 1970. 